\title{
(itis) ENERG
}

Prepared for the U.S. Department of Energy

under Contract DE-AC05-76RL01830

\section{Aggregate Building Simulator (ABS) Methodology Development, Application, and User Manual}

JA Dirks

WJ Gorrissen

November 2011

Pacific Northwest

NATIONAL LABORATORY

Proudly Operated by Battelle Since 1965 


\title{
DISCLAIMER
}

This documentation was prepared as an account of work sponsored by an agency of the United States Government. Neither the United States Government nor any agency thereof, nor Battelle Memorial Institute, nor any of their employees, makes any warranty, express or implied, or assumes any legal liability or responsibility for the accuracy, completeness, or usefulness of any information, apparatus, product, or process disclosed, or represents that its use would not infringe privately owned rights. Reference herein to any specific commercial product, process, or service by trade name, trademark, manufacturer, or otherwise does not necessarily constitute or imply its endorsement, recommendation, or favoring by the United States Government or any agency thereof, or Battelle Memorial Institute. The views and opinions of authors expressed herein do not necessarily state or reflect those of the United States Government or any agency thereof.

\author{
PACIFIC NORTHWEST NATIONAL LABORATORY \\ operated by \\ BATTELLE \\ for the \\ UNITED STATES DEPARTMENT OF ENERGY \\ under Contract DE-AC05-76RL01830
}

Printed in the United States of America
Available to DOE and DOE contractors from the Office of Scientific and Technical Information, P.O. Box 62, Oak Ridge, TN 37831-0062; ph: (865) 576-8401, fax: (865) 576-5728 email: reports@adonis.osti.gov

Available to the public from the National Technical Information Service, U.S. Department of Commerce, 5285 Port Royal Rd., Springfield, VA 22161

ph: (800) 553-6847, fax: (703) 605-6900

email: orders@ntis.fedworld.gov

online ordering: http://www.ntis.gov/ordering.htm 
PNNL-19943

\section{Aggregate Building Simulator (ABS) Methodology Development, Application, and User Manual}

JA Dirks

WJ Gorrissen

September 2010

Prepared for

U.S. Department of Energy

under Contract DE-AC05-76RL01830

Pacific Northwest National Laboratory

Richland, Washington 99352 



\section{Summary}

Building energy simulation, through computer modeling, is of growing importance in myriad uses from the design of individual building to the design of national policy. The Building Technologies Program (BTP) has supported the development of EnergyPlus and has made it a requirement that where possible, this tool be used for program planning and analysis. As such, PNNL has adopted EnergyPlus as a tool for examining the medium and long-term impacts of BTP program activities.

As the relationship between the national building stock and various global energy issues becomes a greater concern, it has been deemed necessary to develop a system of predicting the energy consumption of large groups of buildings. Ideally this system is to take advantage of the most advanced energy simulation software available, be able to execute runs quickly, and provide concise and useful results at a level of detail that meets the users' needs without inundating them with data. For program planning and evaluation purposes it is desirable for this system to be able to model the consumption of the following groups of buildings:

- The stock of buildings as they currently exist

- The current stock of buildings assuming one or more changes (e.g., replace all windows with high performance windows) to all or a portion of the stock

- New buildings built to a common level of performance (e.g., the current energy code)

- New buildings built with a higher level of performance (e.g., $30 \%$ or $50 \%$ above code)

While EnergyPlus is detailed, accurate, and the premier building energy simulation tool, it is difficult to use it to rapidly simulate large numbers of buildings. The tool outlined in this report was developed in an effort to overcome the challenges hindering the use of EnergyPlus in large-scale, multibuilding simulations. To develop this modeling system three tools were used, the output of each becoming the input for the next, these tools were:

- GParm, a mail merge utility

- National Renewable Energy Laboratory Preprocessor, an application programming interface for EnergyPlus

- Energy Plus, a whole building energy simulation program

The interaction between these tools and the organization of the resulting outputs is controlled through the use of various utilities in a Linux environment. This modeling system is known as the Aggregate Building Simulator (ABS).

The resulting methodology that was developed allows the user to quickly develop and execute energy simulations of many buildings simultaneously, taking advantage of parallel processing to greatly reduce total simulation times. The result of these simulations can then be rapidly condensed and presented in a useful and intuitive manner.

To test this methodology and demonstrate its effectiveness, a simulation making use of all available functionality was performed. This test consisted of a large group of buildings with diverse 
characteristics, required a custom report variable set, and the building characteristics that were simulated that were beyond the current functionality of the preprocessor.

The building characteristic that was selected for consideration was window type, specifically, comparing a set of buildings having energy star windows (with properties varying to meet the requirements for their climate) with four other sets of buildings, all with high performance electrochromic (EC) windows. EC windows were selected for use in the test simulation because of their complexity, the preprocessor's inability to handle the required control types, and to demonstrate the text substitution capability.

This report, and the operation of ABS, assumes that the reader is already conversant with:

- EnergyPlus modeling and the requirements for executing that model and interpreting the results. (For more information on the use of EnergyPlus see the link to the User's Manual listed in the references.)

- Linux syntax and basic commands. 


\section{Contents}

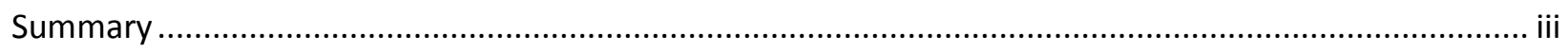

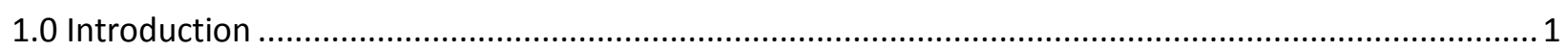

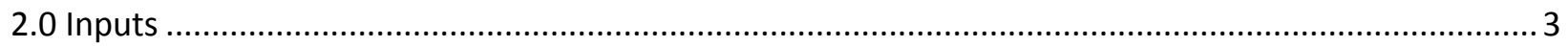

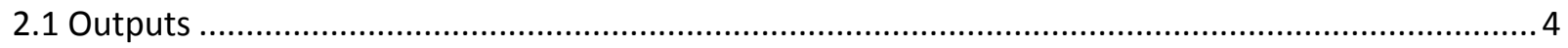

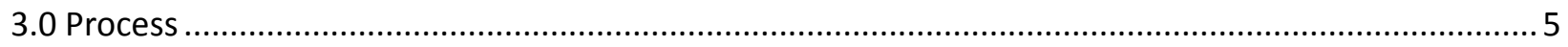

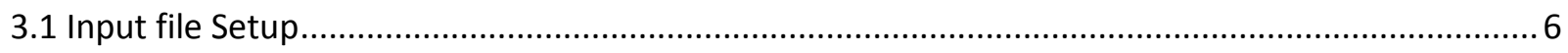

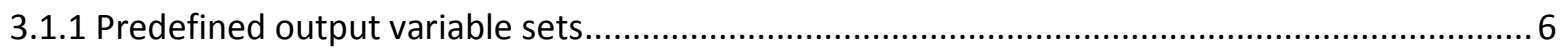

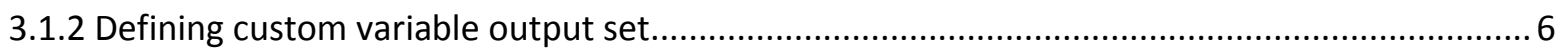

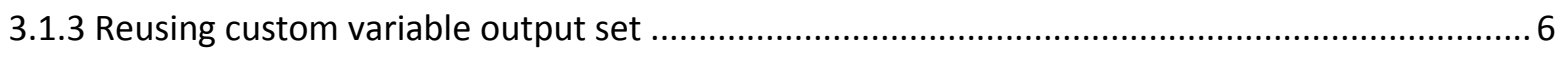

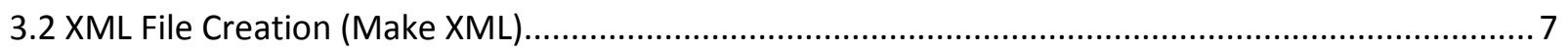

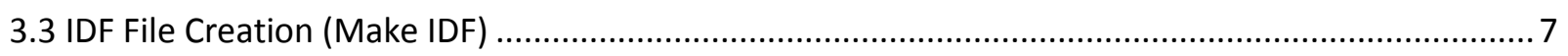

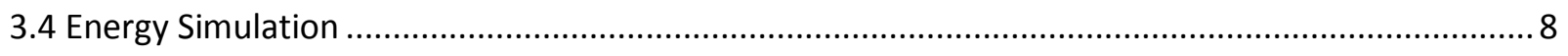

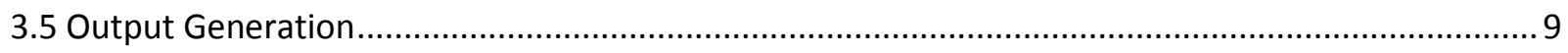

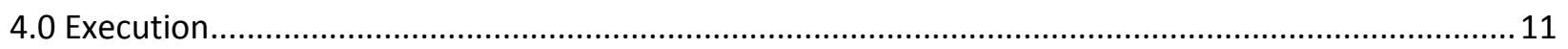

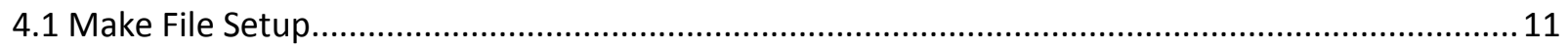

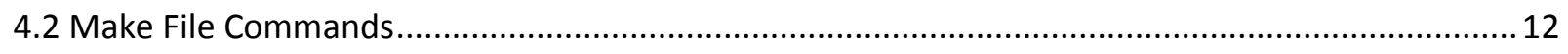

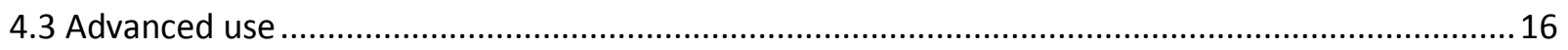

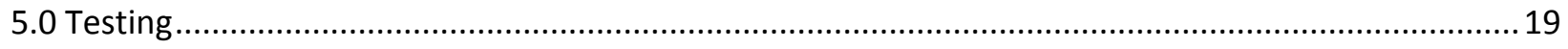

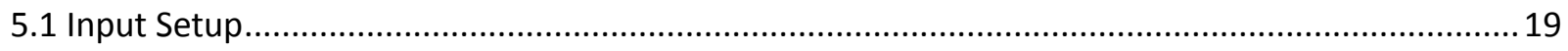

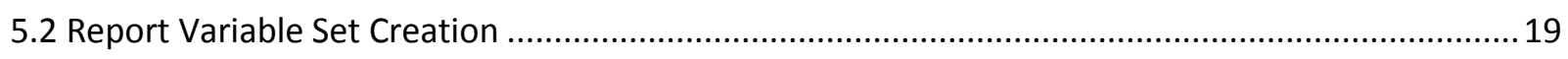

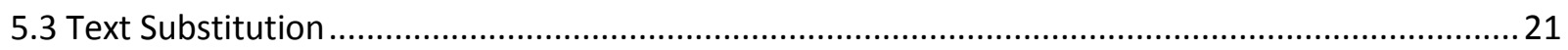

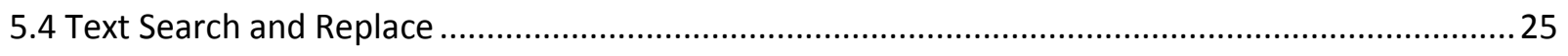

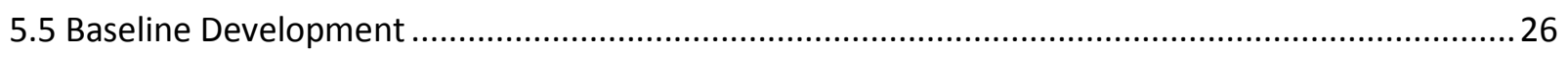

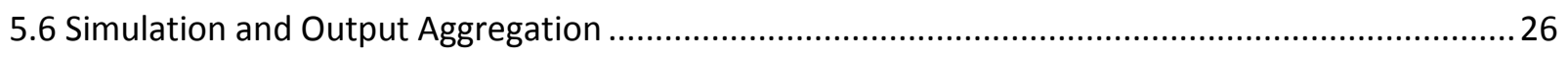




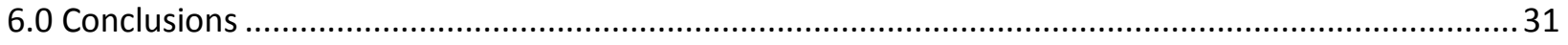

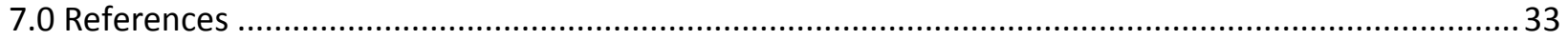

Appendix A: Aggregate Building Simulator Input Generator ........................................................... A-1

Appendix B: Aggregate Building Simulator Input Variables, Description, Default Values ........................ B-1

Appendix C: Aggregate Building Simulator Allowable Footprint Shapes............................................ 


\section{Figures}

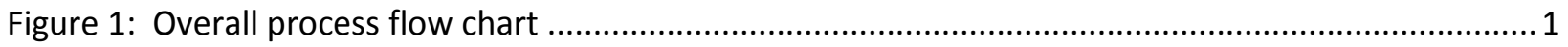

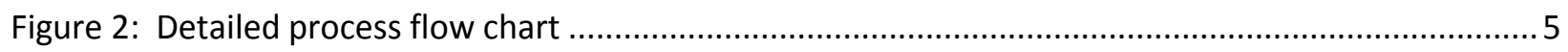

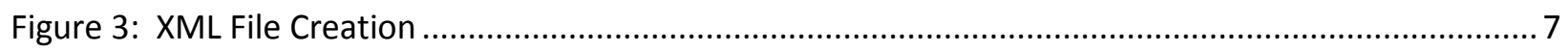

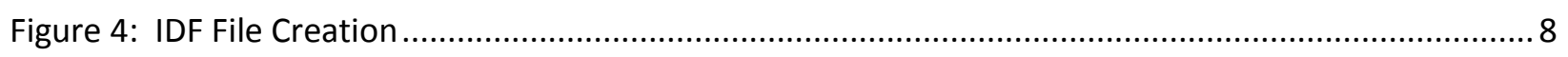

Figure 5: Sample of typical energy plus meter output files ........................................................... 9

Figure 6: Sample of typical ABS summary output.......................................................................

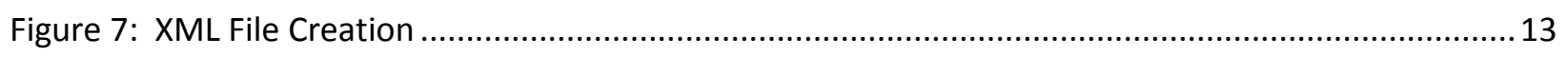

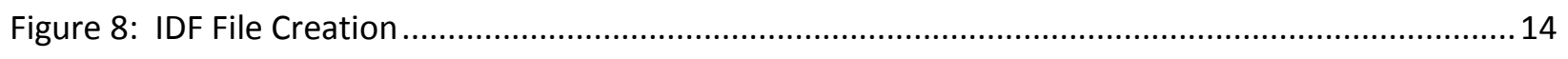

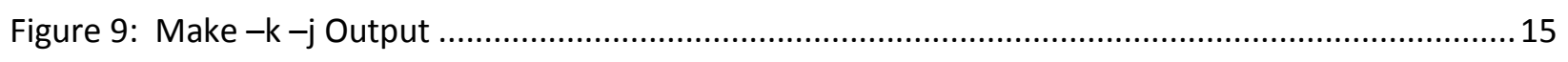

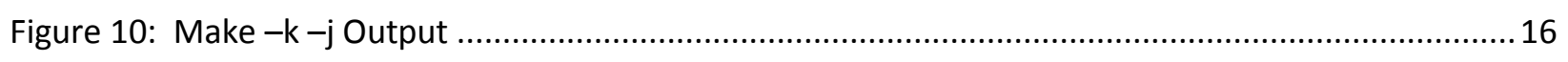

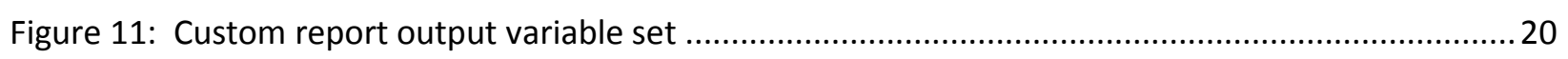

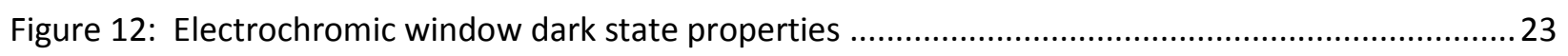

Figure 13: Shading control objects used to model electrochromic windows.......................................24

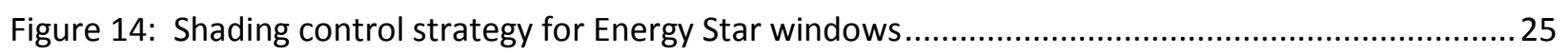

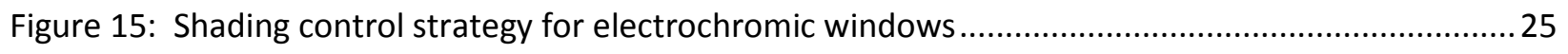




\section{Tables}

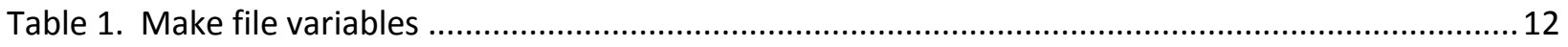

Table 2: EERE 2020 Electrochromic Window Technical Targets ..........................................................2 20

Table 3: Electrochromic window properties for light and dark state ..................................................2 21

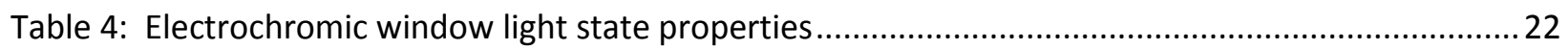

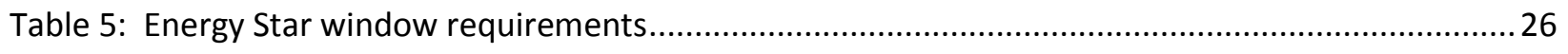

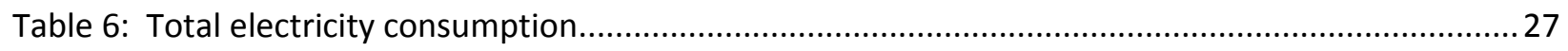

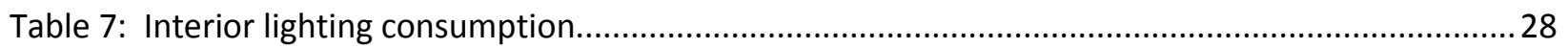

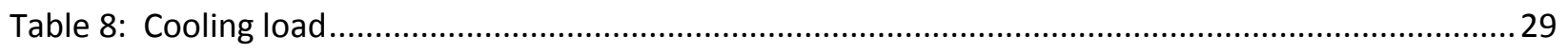

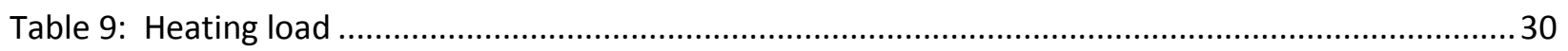




\subsection{Introduction}

EnergyPlus is a powerful tool developed to meet the needs of a diverse group of end users. It is widely considered to be the premier tool for building energy simulation. An EnergyPlus input file can represent an almost infinite number of combinations of building characteristics. The depth and breadth of the inputs give EnergyPlus the flexibility to be usefully applied to a wide range of detailed projects, it also makes the software difficult to use without an in-depth knowledge of the program and the building that the user wishes to represent. In addition, with input files easily reaching thousands of lines in length, the process of putting together a simulation for even a single building is time consuming and has a high potential for error.

The complexity and detail of the EnergyPlus algorithms also contribute to long simulation times and vast quantities of output information. A typical building will generally take about 5 minutes to simulate and will produce many thousands of lines of output text spread across multiple files.

All of the aforementioned issues do not present much of a problem when only a small number of simulations are being performed. If, on the other hand, one wishes to run a large scale parametric building simulation, things quickly become unwieldy. When doing an analysis requiring the simulation of many different types, sizes, and vintages of buildings in a variety of different climate zones the process can become cumbersome with total simulation times start to run into the days. Additionally, even the most powerful desktop computers and business productivity software quickly grind to a halt when dealing with thousands of output files that are produced from which the data needs to be aggregated.

As part of the impacts analysis project, the Building Technologies Program needed an approach to regional and national analysis using EnergyPlus. As such, ABS was developed to overcome the three linked issues that arise from multiple building EnergyPlus simulations (input complexity, long simulation times and post processing massive amounts of data), while taking advantage of a number of separate tools and techniques ( Figure 1). This methodology reduces the required inputs to a single file containing multiple series' of variable values, each representing a single building. This single input then drives a multi threaded simulation in a Linux environment, drastically reducing the total time required. The methodology further capitalizes on the powerful command line tools available in the Linux environment to quickly aggregate the multiple simulation outputs into a single file allowing for useful comparison of outputs across individual buildings.

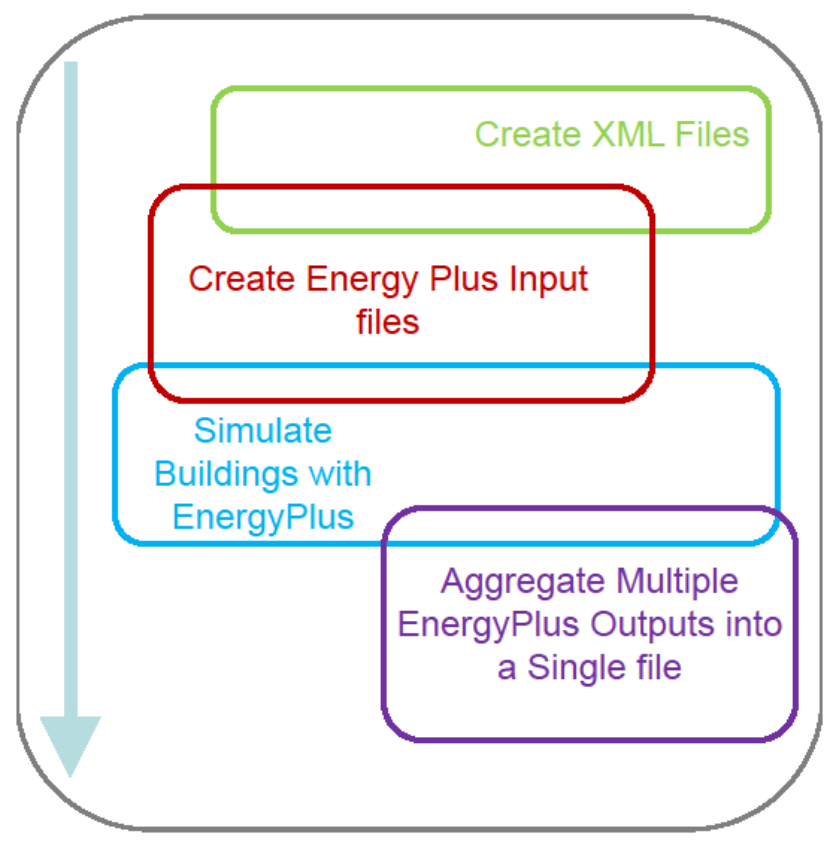

Figure 1: Overall process flow chart 
To test this methodology and demonstrate its effectiveness, a simulation making use of all available functionality was performed. This test consisted of a large group of buildings with diverse characteristics, required a custom report variable set, and the building characteristics that were simulated that were beyond the current functionality of the preprocessor.

Chapter 2 describes the five categories of inputs to ABS: simulation parameters, building attributes, constructions, loads, and systems. Chapter 3 explains the ABS process from input file setup to output generation while model execution and use is laid out in Chapter 4 . Chapter 5 then describes a test of the methodology consisting of a large group of buildings with diverse characteristics that required a custom report variable set and employed some building characteristics that were beyond the current functionality of the XML preprocessor. Lastly, Chapter 6 provides some conclusions from the development and use of ABS. While not essential, a working knowledge of EnergyPlus and Linux are valuable in understanding this report and the operation the Aggregate Building Simulator (ABS). 


\subsection{Inputs}

All the simulated buildings are represented in a single, comma separated, text (CSV) file. Each line in the input file represents a single building and creates a single EnergyPlus input file. As currently configured, there are up to 709 variable values that the user can easily define for each building. Other than the requirement that each building have the same number of variables defined, there is no lower limit with respect to how many values are used to describe a building. If a variable is not defined, it is set to a reasonable value, inferred based on the other user inputs for that specific building, using the Aggregate Building Simulator (ABS) Input Generator (see Appendix A). The input file contains, in general, variables related to five different aspects of the simulation (see Appendix B for a complete listing of all inputs):

- Simulation parameters

- Define how the simulation is carried out, including: simulation timestep, simulation run period, convergence tolerances, and others.

- Building attributes

- Control shape and size of the building and include: floor area, number of stories, footprint shape, rotation, location, and others.

- Constructions

- Define the fabric of the building, either layer by layer or using single layer theoretical materials. All energy related properties can be defined for windows, walls, roofs, and floors.

- Loads

- Internal loads (people, lighting, and miscellaneous) can be defined for each building. The loads are considered to be located uniformly throughout each building. Power density, sensible ratio, heat to space and fuel type (electric or gas) can be defined for two types of miscellaneous load.

- Systems

- The system variables control the attributes of the heating, ventilation, and air conditioning (HVAC) and domestic hot water (DHW) systems. The DHW system is defined in terms of fuel, rated capacity, storage volume, and efficiency. For HVAC systems, all sizing is handled by EnergyPlus, but the user can define system type, fuel type and the efficiency of the various components.

In addition to the CSV input file that defines the building characteristics, a GPARM/XML ${ }^{1}$ template file is also needed to create the individual EnergyPlus input files. This template file contains all of the default variable values and serves to relate the variable to specific objects in the EnergyPlus input

\footnotetext{
${ }^{1}$ Extensible markup language (XML) is simply a standardized way of conveying information and is useful when one wishes to pass information between two pieces of software that use different types of inputs.
} 
files. There is typically no need for a user to modify the template file. However, if a particular functionality is required and is not handled within the current capabilities of the methodology, the template file can be modified to expand these capabilities.

\subsection{Outputs}

The final product of the methodology outlined here is a single file containing an aggregation of all the individual meter.csv files ${ }^{2}$ that are produced by EnergyPlus. The resultant file has the timesteps along the vertical axis and the output variables, grouped by building, along the horizontal axis. The file contains a mixture of units and values collected over varying periods, so care needs to be taken when interpreting the results. See Section 3.5 Output Generation for a more complete description of the output.

\footnotetext{
${ }^{2}$ One of the outputs available within EnergyPlus is meters. A meter is energy consumption associated with a piece of equipment, end-use, or building and can be output at the time step, hourly, monthly, or annually.
} 


\subsection{Process}

The steps to producing the required outputs from the CSV and template files are outlined below in Figure 2. Each of the main steps is shown with the external inputs and outputs shown in green, internal inputs and outputs in blue, and internal processes in yellow. The heavy colored lines show the high level process stages and the colored text, their associated commands. Each step will be examined below in detail.

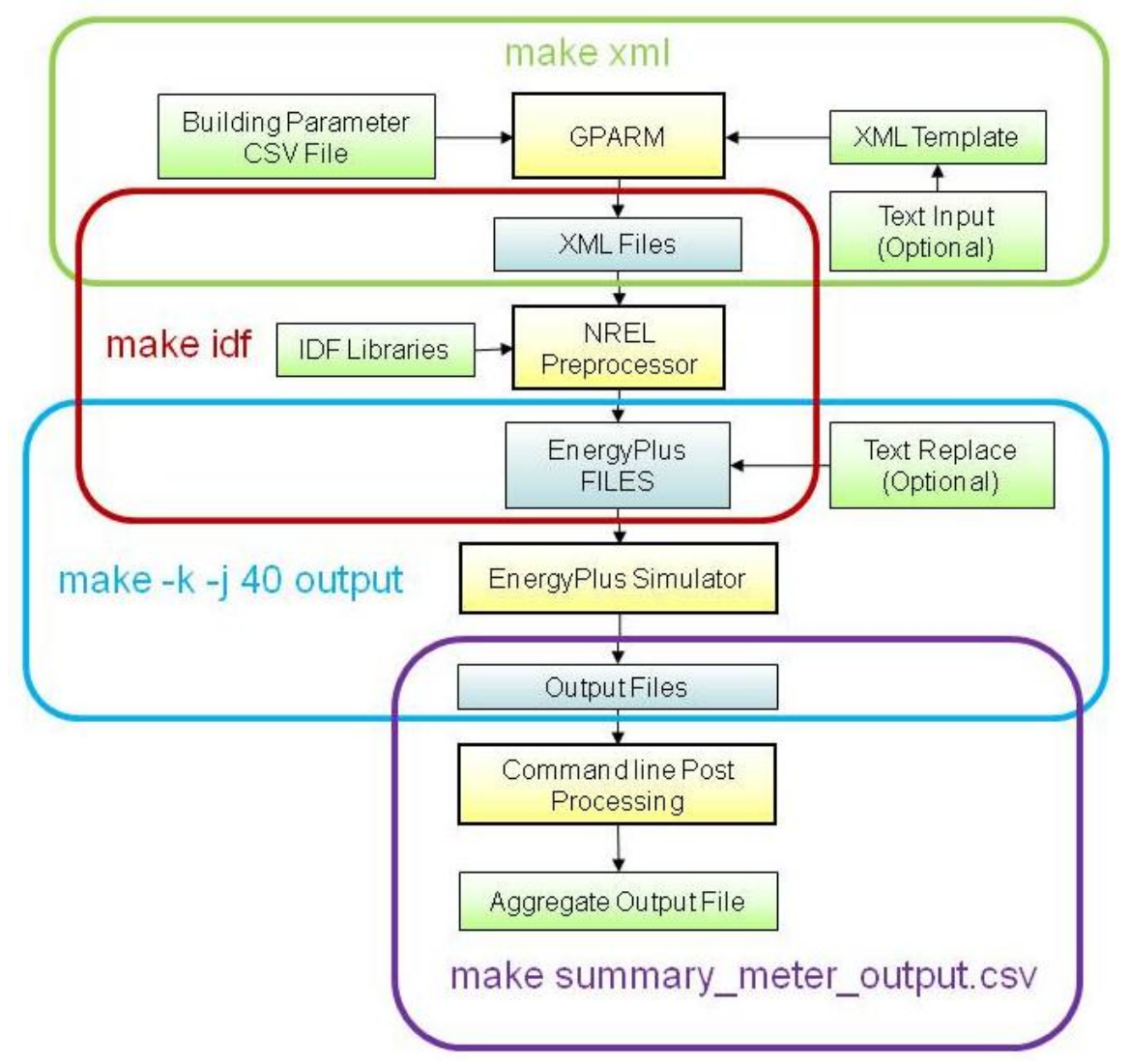

Figure 2: Detailed process flow chart 


\subsection{Input file Setup}

The initial step of the process is to set up the parameter and template files. The parameter file should be filled in with all the variables that the user wishes to parameterize and also with those variables for which default values are not appropriate. This latter category includes any variable that has absolute units such as floor area and number of floors, versus variables that are scaled, such as lighting power density, where using the default values produces more realistic results. To see a description of each variable and its default value see Appendix B.

\subsubsection{Predefined output variable sets}

In order to define which output variables are produced during the simulations, one of the following output variable set names can be entered as the value for the input variable ReportVarSetKey:

- BasicReportsAndABUPS

- BasicReportsAndABUPS_CSV

- ReportVarforDX

- ReportVarforlECstudy

- ReportVarforVAVChillerBoiler

- ReportTestForReader

- BasicReportsAndAPUBS_BEOpt

- ReportsForCBECSAnalyses

- BasicReportsAndABUPS_Benchmark

- ReportsForSmallCBECSmicroCogenAnalyses

- HourlyEnergyOnly

\subsubsection{Defining custom variable output set}

It is possible to create a custom variable set. To create a one-time report variable set, the desired output objects (written in standard EnergyPlus Input Data Format IDF) are placed in the template file between the <subtext> and </subtext> tags. (Any text entered between these tags is passed directly into each IDF file and hence, needs to be entered in proper EnergyPlus syntax.) If new variables have been entered in this manner and the user does not wish to return any other results, the value of ReportVarSetKey should be set equal to Null_Set.

\subsubsection{Reusing custom variable output set}

If the user wishes to create a variable output set that can be reused for other projects, it is possible to create a new output package that can then be called using ReportVarSetKey. To accomplish 
this, the user needs to point to the Macro Control path ${ }^{3}$ and open the HPBReportVariableSets.imf file. The new output variable set should be appended to the existing content of the file in the flowing format:

\#\#def Name_of_variable_set[]

Desired outputs, in proper EnergyPlus Syntax

\#\#enddef

The user can now call the above output variable set by assigning its name (the text between \#\#def and [J) to the ReportVarSetKey variable.

\subsection{XML File Creation (Make XML)}

With the CSV and template files defined, the user can now create a set of XML files that are used to build the required EnergyPlus input files. This is accomplished by using a mail merge utility called GParm to combine the CSV file with the template file, creating one XML file for each row of the CSV file as shown in Figure 3. Each XML file contains the desired characteristics of a single building. The XML files can now be submitted to the NREL preprocessor.

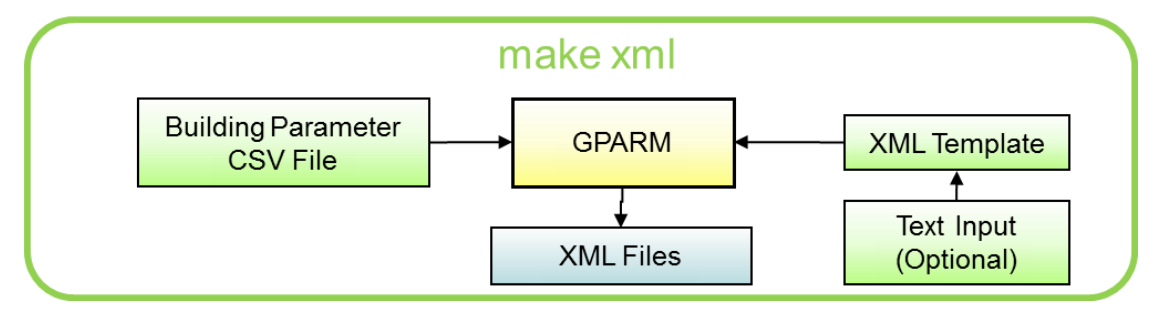

Figure 3: XML File Creation

\subsection{IDF File Creation (Make IDF)}

Developed by NREL as a tool to simplify the process of interfacing with the EnergyPlus simulation engine, the preprocessor is described in the documentation as:

... a computer program that reads an abstract description of a building and writes a complete, fully-functional EnergyPlus input file. The building XML model is the text input file to the preprocessor, contains a minimal set of high-level parameters to describe the building type, geometry, loads, and systems. The preprocessor constructs the resulting EnergyPlus building

\footnotetext{
${ }^{3}$ The macro control path points to the directory in which key files used over the course of the methodology are stored. Typically these files are located wherever the NREL (National Renewable Energy Laboratory) preprocessor (a key element of the methodology) is saved, under /bin/include/.
} 
model by automatically generating and connecting all of the required EnergyPlus objects based on a set of built-in modeling assumptions. (NREL 2008)

The preprocessor essentially allows the user to define only those attributes that are related to a building's energy performance, automatically generating the IDF language required by EnergyPlus. By driving the preprocessor inputs using a CSV file and an XML template, the Aggregate Building Simulation (ABS) tool further reduces the required expertise of the user and makes it possible to easily generate the multiple building input files required for large-scale parametric runs as shown in Figure 4 . To further facilitate the production of building attribute input files, a CSV input generator was developed using PNNL internal funding. A short description of the input file generator is available in Appendix A.

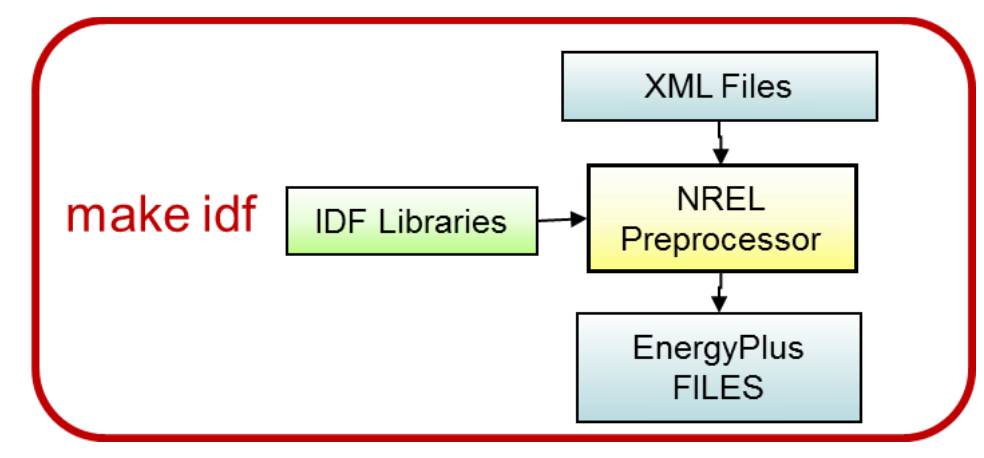

Figure 4: IDF File Creation

\subsection{Energy Simulation}

All of the actual energy modeling is performed using EnergyPlus. According to the documentation:

EnergyPlus is an energy analysis and thermal load simulation program. Based on a user's description of a building from the perspective of the building's physical make-up, associated mechanical systems, etc., EnergyPlus will calculate the heating and cooling loads necessary to maintain thermal control setpoints, conditions throughout an [sic] secondary HVAC system and coil loads, and the energy consumption of primary plant equipment as well as many other simulation details that are necessary to verify that the simulation is performing as the actual building would. (EnergyPlus 2010)

The complexity of the EnergyPlus inputs and the need to perform many simultaneous simulations was a key driver behind the development of ABS. With the ABS modeling system, the user does not directly interact with EnergyPlus in any way other than setting the simulation parameters in the CSV input file. The methodology also does not require any previous knowledge of EnergyPlus although it is extremely useful when modeling something outside the capability of the preprocessor. 


\subsection{Output Generation}

Following the EnergyPlus simulations all outputs are aggregated into a single output CSV file. Each row in all the output files (representing the results for a particular timestep) is combined together, and the results are then collected into a single file, as shown in Figure 5 and Figure 6.

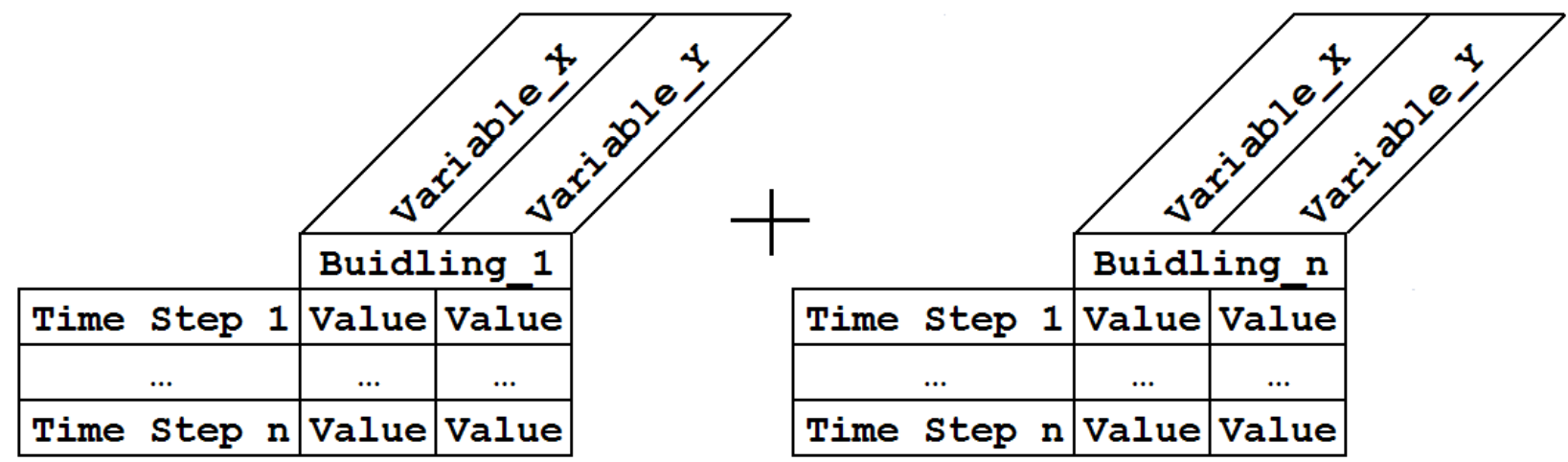

Figure 5: Sample of typical energy plus meter output files

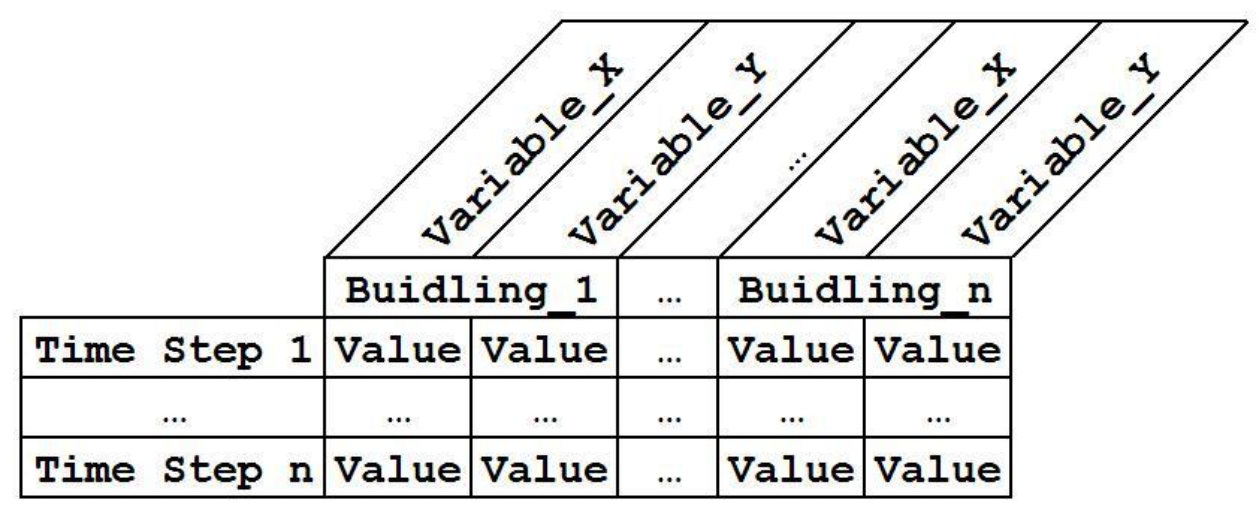

Figure 6: Sample of typical ABS summary output 


\subsection{Execution}

With each of the required inputs developed, the user can now follow the detailed execution steps laid out below. The process execution can be restarted from the beginning of any of the main steps.

\subsection{Make File Setup}

To control the execution of each of the processes outlined in Section 3 and to ensure the methodology is flexible and simple to execute, Make (a software development utility) is used to control execution order and file dependencies. Make was originally developed to compile executable files from chains of interdependent source files. For the purpose of executing the methodology outlined here, the Make utility is used in a similar fashion to control the dependencies between the various input and output files as well as to control the required Linux commands. Make file dependencies work based on the file timestamps. If a file is changed, any files that depend on that changed file are, in turn, remade. This ensures that only files that have been changed are re-run, either through the preprocessor or EnergyPlus. Because all XML files are dependent on a single CSV file, this system breaks down if the XML files are remade; every XML file will have a new timestamp and so the entire process will be re-run. Care should be taken to not unnecessarily rebuild the XML files because this could lead to many hours of unnecessary simulation of unchanged files ${ }^{4}$.

\footnotetext{
${ }^{4}$ If changes need to be made to only a small number of the XML files, the best approach is to delete from the CSV file all but the rows corresponding to those XML files (leaving the row ID numbers unchanged). Rerunning the entire methodology with the reduced CSV will only simulate the buildings of interest.
} 
To ensure flexibility, the first portion of the Make file defines a number of variables, as shown in Table 1. Any changes that a user needs to make to set up a new run will occur in these variables.

Table 1. Make file variables

$\begin{array}{ll}\text { Variable } & \text { Function } \\ \text { EXCELXML_DIR } & =\text { Project Directory } \\ \text { GPARM } & =\text { Path to GParm Script } \\ \text { RUNEPLUS } & =\text { Path to Energy Plus } \\ \text { PPROC } & =\text { Path to preprocessor } \\ \text { XMLFILE_DIR } & =\text { XMLFile Directory } \\ \text { INPUT_DIR } & =\text { IDF File Directory } \\ \text { OUTPUT_DIR } & =\text { EnergyPlus Output Directory } \\ \text { TEMPLATE_FILE } & =\text { Name of template file } \\ \text { PARM_FILE } & =\text { Name of parameter file }\end{array}$

\subsection{Make File Commands}

Once the variables have been defined and the Make file saved to the project directory (under the name "makefile", with no extension or quotes) it is executed with the following steps:

1. Create XML files (Figure 7). This is executed as a separate step because all of the XML files created depend on a single input file, therefore if the XML files are remade, all the timestamps will be updated and all simulations will be rerun. The command make XML executes the following:

$\$(G P A R M)-p \$\left(P A R M \_F I L E\right)-t \$\left(T E M P L A T E \_F I L E\right)-d \$\left(X M L F I L E \_D I R\right)-f$ 'Building_\{\}.XML' $-n 8$

- All variables areas defined as above

- $-\mathbf{p},-\mathbf{t}$, and $-\mathbf{d}$ indicate the parameter file, the template file, and the destination directory, respectively.

- -f 'Building_\{\}.XML' controls the naming of the output files, in this case the braces, \{\} , are replaced by the ID number (value in first column) of the corresponding row in the CSV file, creating Building_1.XML to Building_n.XML

- $\quad-\mathbf{n} \mathbf{8}$ causes the process to run on eight cores on a single node; it is typically not useful to run GParm on more than one node because the data transfer times become longer than the processing times. 


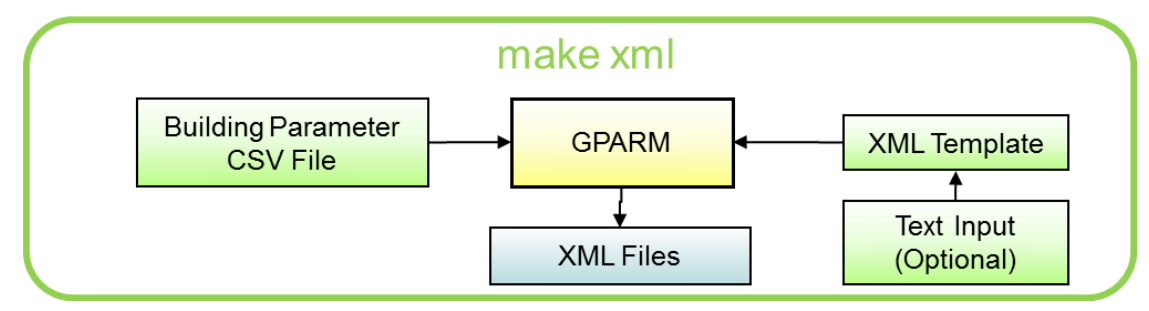

Figure 7: XML File Creation

2. Execute all remaining commands. The command make all runs the preprocessor, executes the EnergyPlus runs, and aggregates the results by running the following lines of code:

make idf (Figure 8)

./input/\%.idf: ./XMLFiles/\%.XML

$\$(P P R O C) \$<\$$

- These lines check if any file within the dependency has been modified and runs the preprocessor if any changes are detected.

- The first line is the make command that is called to execute this step; the user could enter this rather than make all if he or she wished to execute one step at a time.

- The second line defines the file dependencies, using the $\%$ as a wild card to represent all files that have the appropriate extension. In this case, the IDF files from the input directory depend on the XML files from the XMLFiles directory.

- The third line is the command that is executed for each input (XML) file. PPROC is the variable representing the directory where the preprocessor is located and the command to run it. The '< 'symbol is used to represent the input file, the XML file in this case, and the '@' represents the target file, the IDF file in this case. In a Make file the '\$' symbol is used before calling variables. 


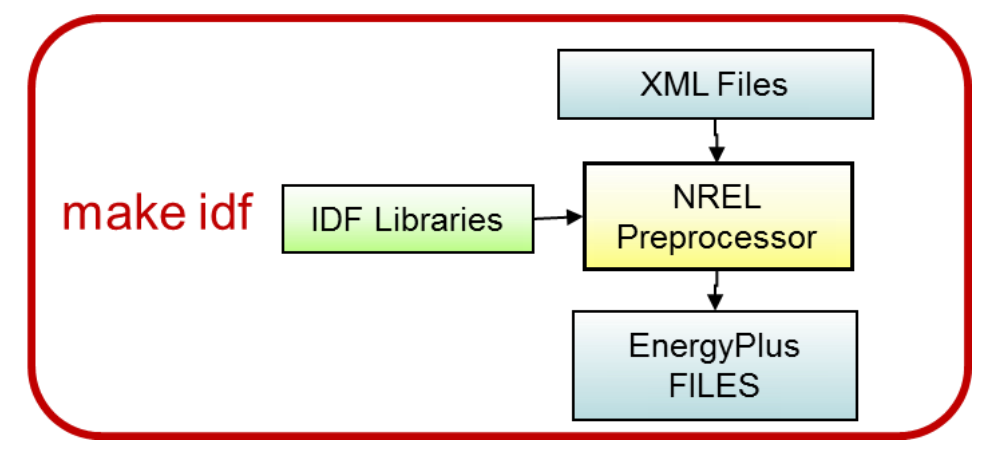

Figure 8: IDF File Creation

make - $k$-j 40 output (Figure 9)

\$(OUTPUT_DIR)/\%.meter.CSV: \$(INPUT_DIR)/\%.idf

@/tools/bin/qwait $\$(R U N E P L U S)$-o $\$^{*}-i \$\left(I N P U T \_D I R\right)-p$ S(OUTPUT_DIR) 'basename $\$<'$

- These lines check if any file within the dependency has been modified and runs EnergyPlus if any changes are detected.

- This first line is the make command associated with this step. The $-\mathrm{j}$ indicates that the number following controls how many processers should be made available to run separate instances of EnergyPlus. This number is limited by the properties of the cluster being used. The $-\mathbf{k}$ ensures that the whole process will continue even if errors occur.

- The second line defines the file dependencies; in this case the output '.meter.CSV' files depend on the IDF files.

- The third line is the command that executes EnergyPlus as needed. @/tools/bin/qwait runs the command through the clusters queuing system, as dictated by the value following $-j$ in the make command. $-\mathbf{o} \mathbf{\$}$ ( controls the output file name, in this case taking the same name as the input file. $-\boldsymbol{i}$ and $-\boldsymbol{p}$ control the input and output file paths, respectively. 'basename $\$<$ removes the path from the input (IDF) file leaving only the file name to be passed to runeplus ${ }^{5}$ as the input file.

\footnotetext{
${ }^{5}$ Runeplus is a script developed to facilitate running EnergyPlus in the Linux environment.
} 


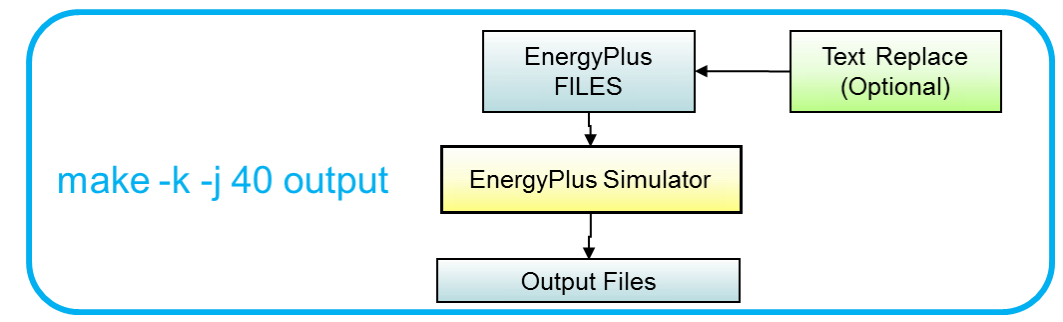

Figure 9: Make $-\mathrm{k}-\mathrm{j}$ Output

make summary_meter_output.CSV (Figure 10)

cd./output

find Building_?.meter.CSV | sort -t_- $2,2-n$ | xargs paste $-d,>$../summary_meter_output_1.CSV

find Building_??.meter.CSV | sort $-t \_-k 2,2-n$ | xargs paste $-d,>$../summary_meter_output_2.CSV

find Building_???.meter.CSV | sort -t_ $-k$ 2,2 -n / xargs paste $-d,>$../summary_meter_output_3.CSV

find Building_1???.meter.CSV | sort -t_- 2,2 -n | xargs paste $-d,>$../summary_meter_output_4.CSV

find Building_2???.meter.CSV | sort -t_- $2,2-n$ | xargs paste $-d,>$../summary_meter_output_5.CSV

find Building_3???.meter.CSV | sort -t_- $-k$ 2,2 -n | xargs paste $-d,>$../summary_meter_output_6.CSV

find Building_4???.meter.CSV | sort -t_- $2,2-n$ | xargs paste $-d,>$../summary_meter_output_7.CSV

find Building_5???.meter.CSV | sort -t_- $2,2-n$ | xargs paste $-d,>$../summary_meter_output_8.CSV

find Building_6???.meter.CSV | sort -t_- $2,2-n$ | xargs paste $-d,>$../summary_meter_output_9.CSV

find summary_meter_output_?.CSV | sort -t_-k 4,4 -n | xargs paste -d , > ./summary_meter_output.CSV sed $-i$ 's/,DateVTime,/,/g' summary_meter_output.CSV

sed $-i$ 's/, ..V... ........,/,/g'summary_meter_output.CSV

- These lines aggregate all of the individual outputs into a single file containing all of the simulation results.

- The first line is the make command that initiates this portion of the Make file

- The second line (cd ./output) changes the current directory to the output directory.

- Lines 3 through 11 combine the output files by combining each of the rows. find Building_?.meter.CSV produces a list of the output files where the name matches the pattern Building_?.meter.CSV. The list is then passed as an argument to sort $\mathbf{- t}-\mathbf{- k} \mathbf{2 , 2}-\mathbf{n}$, which puts the files in numerical order and passes them to xargs paste $-\mathbf{d}$, which does the final concatenation into the intermediate summary file summary_meter_output_n.CSV.

- Line 12 concatenates together the results on lines 3 through 11 to produce a final summary file containing the data for every output file. This is accomplished in the same manner as above using find, sort, and paste. 
- The last two lines remove the timestamp from all but the first column by using sed to find and delete the desired entries.

- This method is currently limited to simulations of less than 7000 buildings; to increase this capability, simply copy the $11^{\text {th }}$ line (find Building_6???) and paste it directly underneath itself. Add 1 to the building designation number (Building_6??? Becomes Building_7???) and the intermediate summary file number (summary_meter_output_9.CSV becomes summary_meter_output_10.CSV).

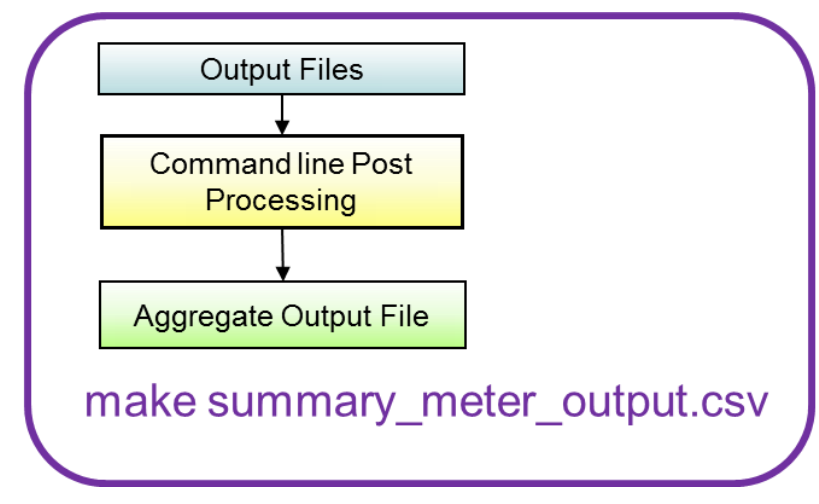

Figure 10: Make -k-j Output

\subsection{Advanced use}

As was mentioned above, it is possible to directly pass pieces of IDF building description from the template file through to each IDF file. This is used to simulate building systems that fall outside the capabilities of the preprocessor. This is an advanced capability that requires the user to be proficient in the use of EnergyPlus and to have a basic understanding of regular expressions ${ }^{6}$, the Linux command line interface, and the sed (Linux stream editor) utility.

Generally there are two parts to increasing the simulation capabilities in this way. First, the user must create the required EnergyPlus objects in the proper IDF format and place the objects between the $<$ subtext $>$ and </subtext $>$ tags in the template file. The objects are included in addition to any output variable objects that the user has placed between the tags; the order of the objects has no impact on the final results.

The next step occurs after the preprocessor is run, creating the IDF files, but before the actual EnergyPlus simulation. To access the files before EnergyPlus runs, it will be necessary to enter the

\footnotetext{
${ }^{6}$ Regular expressions provide a means to search not only based on the content of text, but also based on the location and arraignment of text. As well one can also use regular expressions to match types or ranges of charters.
} 
individual make commands (make idf, make -k -j 40 output, and make summary_meter_output.CSV) rather than make all. After the IDF files have been made using make idf, the user must modify the files to make use of the objects passed through from the template file. This usually involves assigning the new objects to some element of the IDF that are created by the preprocessor, typically by using the sed command to search for a particular string or regular expression and replace it with another. The sed command generally takes the following format:

\section{sed $-i$ 's/string to find/string to replace/g'}

The user must take care to choose a search string that will not capture more than intended, as well the user must understand that the search string is actually a regular expression and any metacharacters must be proceeded by a back slash ( $($ ). In certain instances, the user may wish to replace one entire, preprocessor created object with a user created one; in this case, sed is used as well by leaving the "string to replace" portion of the command blank and placing the entire block of text that needs to be deleted in the "string to find" portion. After applying any user created object(s) as needed, the simulation is continued with the make $-k-j 40$ output command; care should be taken not to use the make all command because all XML files will be remade and changes will be lost. 


\subsection{Testing}

To test the methodology outlined above, a simulation making use of all available functionality was required. This test needed to consist of a large group of buildings with diverse characteristics, require a custom report variable set, and some building characteristics would need to be simulated that were beyond the current functionality of the XML preprocessor.

\subsection{Input Setup}

To execute this test, a set of 3,360 buildings was selected for simulation. The CSV file was populated with building input parameters selected to approximate buildings with the following characteristics:

- Age: 7 vintages: from pre-1946 to present

- Size: 6 floor areas:

- Commercial: less than 5,000 $\mathrm{ft}^{2}$ to greater than $100,000 \mathrm{ft}^{2}$

- Residential': generally less than $1000 \mathrm{ft}^{2}$ to greater than $4000 \mathrm{ft}^{2}$

- Use type: 16 building types, 11 commercial and 5 residential

- Climate: the five climates zones representing $79 \%$ of recent building construction (Belzer 2010).

To capture building heating and cooling loads irrespective of the technology used, and facilitate comparison between buildings, the HVAC system selected for all buildings uses district heating and district chiller water distributed throughout the building via air handling units.

\subsection{Report Variable Set Creation}

Because none of the existing output variable sets reported only district heating, district chiller water, and electricity use, a custom report set was created in the HPBReportVariableSets.imf file, as shown in Figure 11. The ReportVarSetKey variable was then set equal to the name given to this variable set, HourlyEnergyAndPurchWaterOnly.

\footnotetext{
${ }^{7}$ Residential size categories are different for each type of dwelling; the numbers listed here are approximate
} 


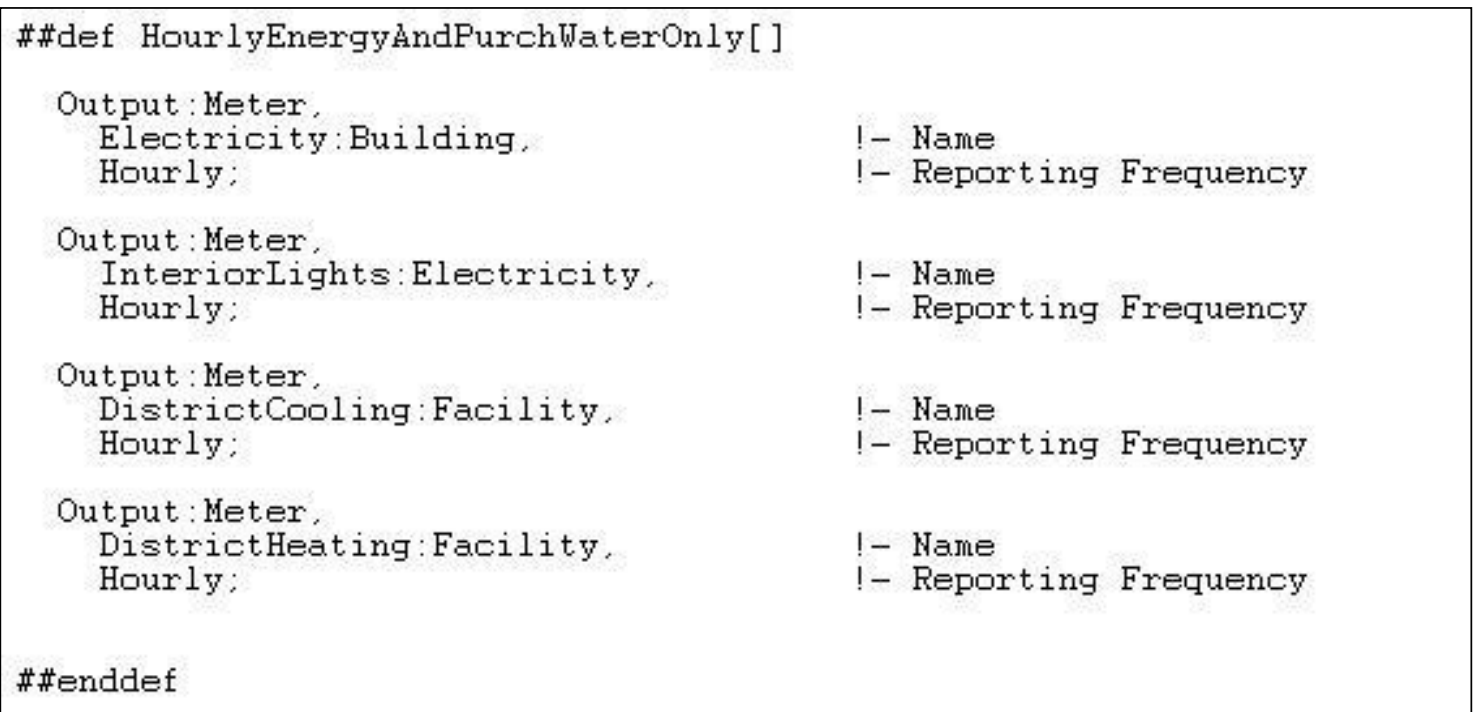

Figure 11: Custom report output variable set

The building characteristic that was selected for variation between simulations was window type, specifically, comparing a set of buildings having energy star windows (with properties varying to meet the requirements for their climate) with four other sets of buildings, all with high performance electrochromic (EC) windows. The four building sets with electrochromic super windows all have windows that meet the EERE 2020 Window Technical Targets in terms of U-value and light/dark VT and SHGC shown in Table 2.

Table 2: EERE 2020 Electrochromic Window Technical Targets

\begin{tabular}{|l|l|l|}
\cline { 2 - 3 } \multicolumn{1}{l|}{} & Light State & Dark State \\
\hline U-Value $\left(B t u / h \cdot \mathrm{ft}^{2} \cdot{ }^{\circ} \mathrm{F}\right)$ & 0.56 & 0.56 \\
\hline SHGC & 0.53 & 0.09 \\
\hline VT & $65 \%$ & $2 \%$ \\
\hline
\end{tabular}

Each building set with EC windows was modeled with different control strategies: 
1. EC always in clear state

2. EC window switch to dark state in response to excessive $(>20)$ discomfort glare index in zone

3. EC windows modulate to dark state when daylighting set point has been reached

4. EC windows switch to dark state either because of excessive $(>20)$ discomfort glare index or modulate until daylighting set point is met.

\subsection{Text Substitution}

EC windows were selected for use in the test simulation because of their complexity, the preprocessor's inability to handle the required control types, and to demonstrate the text substitution capability. The glass properties for the light state EC windows were passed through the preprocessor by first being defined in the CSV input file as a single sheet of theoretical glass. The glass properties were determined using the EnergyPlus object WindowMaterial:SimpleGlazingSystem. This object was new in EnergyPlus 4.0 and allows the user to define a glazing material using only u-value, solar heat gain coefficient (SHGC), and visible transmittance (VT). By simulating a dummy building that had simple glazing systems representing both the EC light and dark states, the detailed properties of an equivalent sheet of glass were determined. Table 3 shows these properties.

Table 3: Electrochromic window properties for light and dark state

\begin{tabular}{|l|l|l|}
\cline { 2 - 3 } \multicolumn{1}{c|}{} & Light State & Dark State \\
\hline Thickness & 0.05469 & 0.05469 \\
\hline Solar Transmittance & 0.45003 & 0.03694 \\
\hline Solar Reflectance Front & 0.38255 & 0.8517 \\
\hline Solar Reflectance Back & 0.38255 & 0.8517 \\
\hline Visible Transmittance & 0.65 & 0.02 \\
\hline Visible Reflectance Front & 0.13254 & 0.23173 \\
\hline Visible Reflectance Back & 0.15003 & 0.43056 \\
\hline IR Transmittance & 0 & 0 \\
\hline Emissivity Front & 0.84 & 0.84 \\
\hline Emissivity Back & 0.84 & 0.84 \\
\hline Conductivity & 0.03411 & 0.03411 \\
\hline
\end{tabular}

The light state windows properties (Table 4) are entered in the CSV because they are considered to be typical window case (and so are applied to the building as any normal window would be). The dark state is considered the altered case so its properties (Figure 12) must be entered separately in the 
text substitution portion of the XML template between the subtext tags in the XML template file. This is because EnergyPlus models any sort of changeable window, including EC windows, to include two descriptions of the window, one in the "open" state, and one in the "closed" state. The open case is assigned to a surface, while the closed case is assigned to a shading control object. The shading control object is assigned to the same surface as the open state window and modulates between the two states based on different inputs. The shading control objects were included in the template file and passed through to each IDF file, as shown in Figure 13.

Table 4: Electrochromic window light state properties

\begin{tabular}{|l|l|}
\hline Variable Name & Variable Value \\
\hline SWinGlass1Name & THEORETICAL GLASS [LIGHT] \\
\hline SWinGlass1Thickness & 0.05469 \\
\hline SWinGlass1SolarTrans & 0.45003 \\
\hline SWinGlass1SolarReflectFront & 0.38255 \\
\hline SWinGlass1SolarReflectBack & 0.38255 \\
\hline SWinGlass1VisibleTrans & 0.65 \\
\hline SWinGlass1VisibleReflectFront & 0.13254 \\
\hline SWinGlass1VisibleReflectBack & 0.15003 \\
\hline SWinGlass1IRTrans & 0 \\
\hline SWinGlass1IREmmFront & 0.84 \\
\hline SWinGlass1IREmmBack & 0.84 \\
\hline SWinGlass1Conductivity & 0.03411 \\
\hline
\end{tabular}




\begin{tabular}{|c|c|}
\hline \multicolumn{2}{|c|}{ 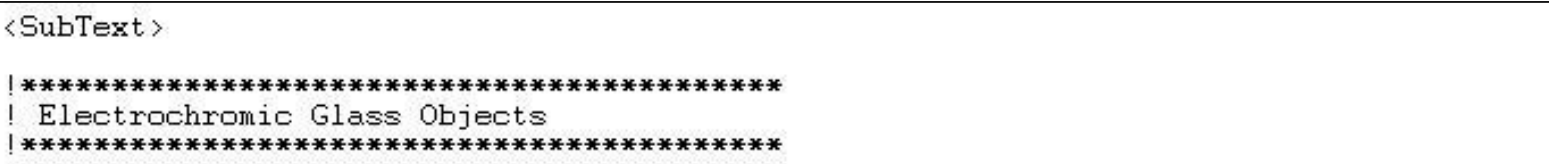 } \\
\hline 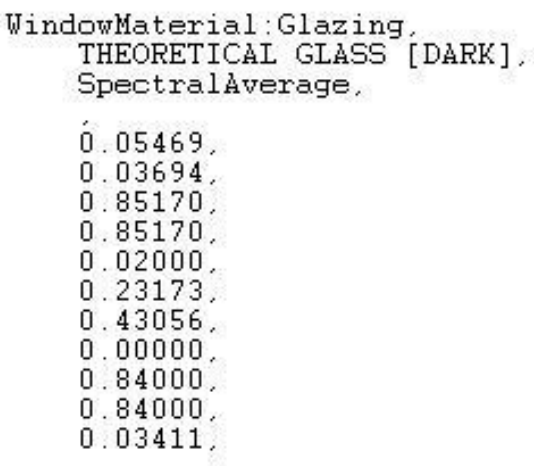 & $\begin{array}{l}\text { !- Name } \\
\text { - Optical Data Type } \\
\text { - Window Glass Spectral Data Set Name } \\
\text { - Thickness \{m\} } \\
\text { - Solar Transmittance at Normal Incidence } \\
\text { - Front Side Solar Ref lectance at Normal Incidence } \\
\text { - Visk Side Solar Ref lectance at Normal Incidence } \\
\text { - Front Side Visible Ref lectance at Normal Incidence } \\
\text { - Back Side Visible Ref lectance at Normal Incidence } \\
\text { - Infrared Transmittance at Normal Incidence } \\
\text { - Front Side Infrared Hemispherical Emissivity } \\
\text { - Back Side Infrared Hemispherical Emissivity } \\
\text { - Conductivity }\{\mathrm{W} / \mathrm{m}-\mathrm{K}\}\end{array}$ \\
\hline $\begin{array}{l}\text { Construction, } \\
\text { Dark State EC Window, } \\
\text { THEORETICAI GLASS [DARK]: }\end{array}$ & $\begin{array}{l}\text { !- Name } \\
\text { ! - Layer } 1\end{array}$ \\
\hline$\cdots$ & \\
\hline$\langle\langle\dot{S}$ ubtext $\rangle$ & \\
\hline
\end{tabular}

Figure 12: Electrochromic window dark state properties 


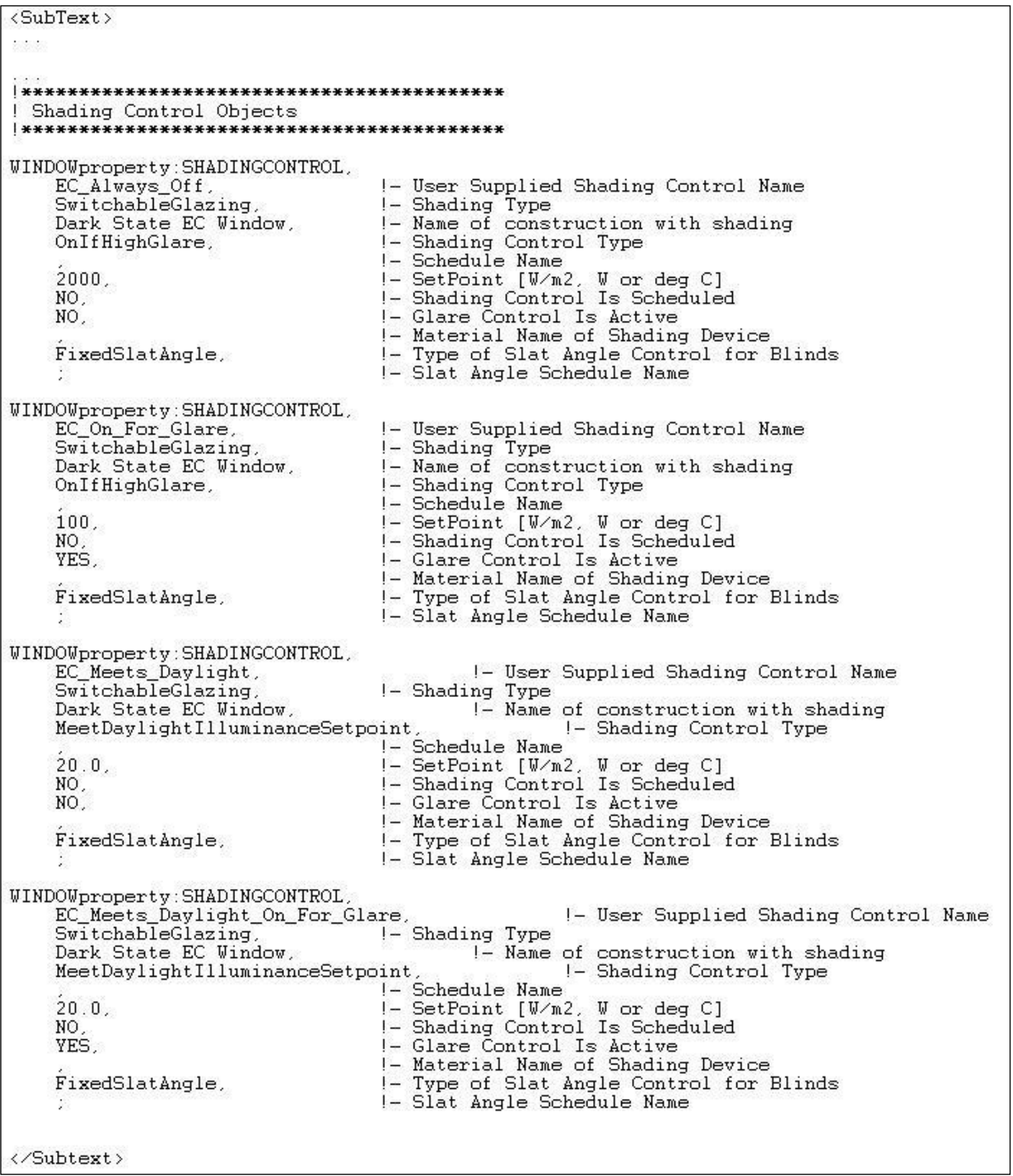

Figure 13: Shading control objects used to model electrochromic windows 


\subsection{Text Search and Replace}

With the CSV and template file prepared, it was then possible to create the XML file with the command make XML and then to create the IDF files with the command make idf. This process was repeated once for each control strategy, ending with four different project directories, each containing a set of 3,360 IDF files. Sed was then used to assign control strategies to the window objects in each IDF; for example, to assign the control strategy of turning the EC window on when high glare is present uses:

sed-i 's/, !-Shading Control Name/EC_On_For_Glare, !-Shading Control Name $/ g^{*}$.idf

to go from the shading control strategy shown in Figure 14 to the strategy shown in Figure 15.

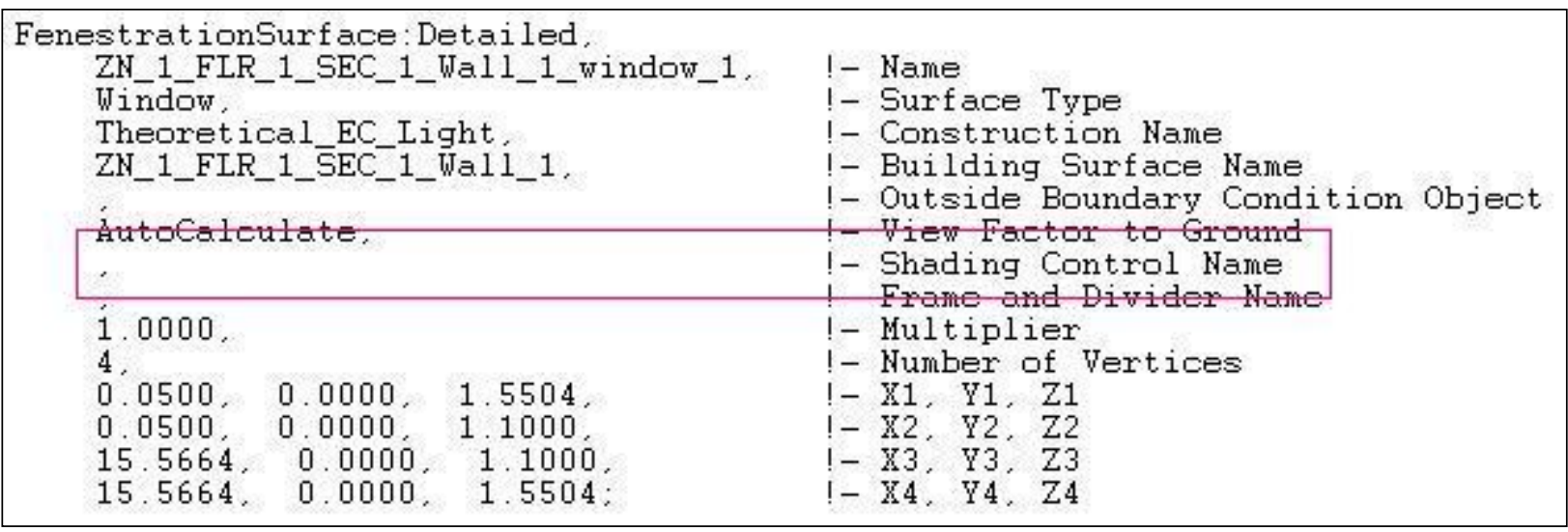

Figure 14: Shading control strategy for Energy Star windows

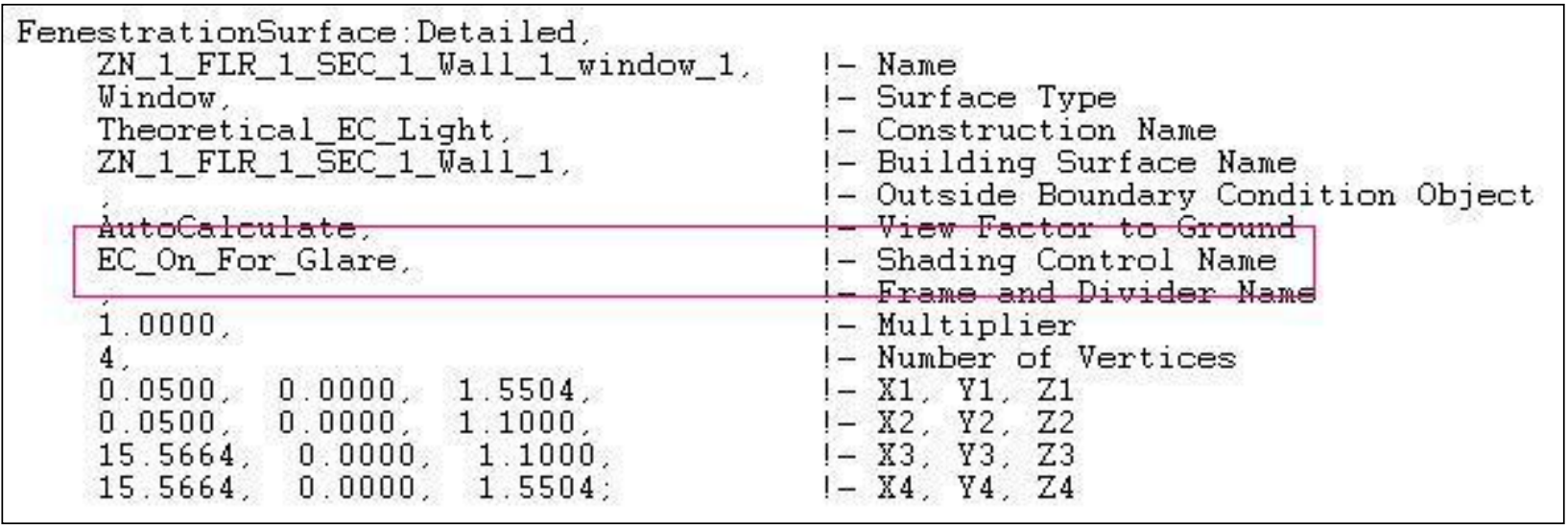

Figure 15: Shading control strategy for electrochromic windows 


\subsection{Baseline Development}

This was repeated for the other two EC control strategies, where it was necessary (no changes were required for the EC always off strategy) to complete the four different sets of IDF files with EC windows. A further set of IDF files was created by assuming that all windows were uncontrolled and met the current Energy Star window requirements for their climate, as shown in Table 5:

Table 5: Energy Star window requirements

\begin{tabular}{|l|l|l|l|}
\hline Climate & U-Value & SHGC & VT \\
\hline Northern & 1.70 & 0.45 & 0.63 \\
\hline North Central & 1.82 & 0.4 & 0.56 \\
\hline South Central & 1.99 & 0.3 & 0.42 \\
\hline Southern & 3.41 & 0.27 & 0.378 \\
\hline
\end{tabular}

\subsection{Simulation and Output Aggregation}

The five sets (one Energy Star baseline set plus four different EC control strategy sets) of IDF files were then run through EnergyPlus (using the make $-k-j 40$ output command) to produce the required outputs for each building.

The outputs were aggregated into a single output summary file using the command make summary_meter_output.CSV. This groups the output files into intermediate summary files of 100 files or less and then combines those intermediate files into one large output file. The intermediate files are necessary to both make the data reasonably easy to work with and to facilitate final output aggregation. For the demonstration run of 3,360 buildings, where each produced four hourly output variables, the resultant summary file contained nearly $1.4 \mathrm{~GB}$ of data. Annual energy use intensity (EUI) results are shown below in Tables 6 through 9. 
Table 6: Total electricity consumption

\begin{tabular}{|c|c|c|c|c|c|c|}
\hline \multirow[b]{2}{*}{ Results Aggregated By: } & \multicolumn{6}{|c|}{ Total Electricity (kBtu/yr/ft ${ }^{2}$ ) } \\
\hline & Baseline & $\begin{array}{c}\text { Baseline } \\
\text { with } \\
\text { daylighting }\end{array}$ & $\begin{array}{c}\text { EC } \\
\text { Always } \\
\text { Off }\end{array}$ & $\begin{array}{c}\text { EC On } \\
\text { For } \\
\text { Glare }\end{array}$ & $\begin{array}{l}\text { EC On For } \\
\text { Daylighting }\end{array}$ & $\begin{array}{l}\text { EC On For } \\
\text { Glare And } \\
\text { Daylighting }\end{array}$ \\
\hline Assembly & 18 & 16 & 15 & 16 & 15 & 16 \\
\hline Education & 26 & 23 & 23 & 24 & 23 & 23 \\
\hline Food Sales & 108 & 104 & 103 & 105 & 103 & 105 \\
\hline Food Service & 88 & 81 & 80 & 83 & 80 & 82 \\
\hline Health Care & 50 & 46 & 46 & 47 & 46 & 46 \\
\hline Lodging & 28 & 26 & 26 & 26 & 25 & 25 \\
\hline Mercantile and Service & 26 & 22 & 21 & 23 & 21 & 23 \\
\hline Office & 27 & 24 & 24 & 25 & 24 & 24 \\
\hline Public Order/Safety & 18 & 15 & 15 & 16 & 15 & 15 \\
\hline Warehouse and Storage & 13 & 12 & 12 & 13 & 12 & 13 \\
\hline Other & 15 & 13 & 12 & 14 & 12 & 13 \\
\hline Single Family Detached & 24 & 22 & 22 & 22 & 22 & 22 \\
\hline Single Family Attached & 120 & 114 & 113 & 116 & 113 & 115 \\
\hline 2 to 4 Unit Buildings & 23 & 22 & 22 & 22 & 21 & 21 \\
\hline 5 or More Unit Buildings & 20 & 18 & 18 & 18 & 17 & 18 \\
\hline Mobile Homes & 46 & 43 & 43 & 44 & 43 & 43 \\
\hline 1945 & 39 & 36 & 36 & 37 & 35 & 36 \\
\hline 1955 & 39 & 36 & 35 & 36 & 35 & 36 \\
\hline 1965 & 41 & 38 & 37 & 38 & 37 & 38 \\
\hline 1976 & 41 & 38 & 38 & 39 & 38 & 38 \\
\hline 1984 & 41 & 38 & 38 & 39 & 38 & 38 \\
\hline 1990 & 44 & 41 & 41 & 42 & 41 & 41 \\
\hline 2000 & 39 & 36 & 36 & 37 & 36 & 37 \\
\hline USA_GA_ATLANTA & 38 & 35 & 34 & 36 & 34 & 35 \\
\hline USA_IL_CHICAGO-OHARE & 39 & 36 & 36 & 37 & 36 & 36 \\
\hline USA_MD_BALTIMORE & 44 & 41 & 41 & 42 & 40 & 41 \\
\hline USA_CA_LOS_ANGELES & 38 & 35 & 34 & 35 & 34 & 35 \\
\hline USA_TX_HOUSTON & 44 & 41 & 41 & 42 & 41 & 41 \\
\hline
\end{tabular}


Table 7: Interior lighting consumption

\begin{tabular}{|c|c|c|c|c|c|c|}
\hline \multirow[b]{2}{*}{ Results Aggregated By: } & \multicolumn{6}{|c|}{ Interior Lights Electricity (kBtu/yr/ft2) } \\
\hline & Baseline & $\begin{array}{c}\text { Baseline } \\
\text { with } \\
\text { daylighting }\end{array}$ & $\begin{array}{c}\text { EC } \\
\text { Always } \\
\text { Off }\end{array}$ & $\begin{array}{l}\text { EC On } \\
\text { For } \\
\text { Glare }\end{array}$ & $\begin{array}{l}\text { EC On For } \\
\text { Daylighting }\end{array}$ & $\begin{array}{l}\text { EC On For } \\
\text { Glare And } \\
\text { Daylighting }\end{array}$ \\
\hline Assembly & 9 & 7 & 6 & 7 & 6 & 6 \\
\hline Education & 10 & 8 & 7 & 8 & 7 & 8 \\
\hline Food Sales & 14 & 11 & 10 & 11 & 10 & 11 \\
\hline Food Service & 24 & 18 & 17 & 19 & 17 & 18 \\
\hline Health Care & 11 & 7 & 7 & 8 & 7 & 7 \\
\hline Lodging & 11 & 9 & 9 & 9 & 9 & 9 \\
\hline Mercantile and Service & 18 & 14 & 13 & 15 & 13 & 15 \\
\hline Office & 10 & 7 & 7 & 7 & 7 & 7 \\
\hline Public Order/Safety & 10 & 7 & 7 & 8 & 7 & 7 \\
\hline Warehouse and Storage & 8 & 7 & 6 & 7 & 6 & 7 \\
\hline Other & 11 & 8 & 7 & 9 & 7 & 9 \\
\hline Single Family Detached & 11 & 10 & 9 & 10 & 9 & 10 \\
\hline Single Family Attached & 55 & 49 & 47 & 50 & 47 & 50 \\
\hline 2 to 4 Unit Buildings & 11 & 9 & 9 & 10 & 9 & 9 \\
\hline 5 or More Unit Buildings & 11 & 9 & 9 & 9 & 9 & 9 \\
\hline Mobile Homes & 11 & 9 & 8 & 9 & 8 & 9 \\
\hline 1945 & 15 & 11 & 11 & 12 & 11 & 12 \\
\hline 1955 & 15 & 12 & 11 & 12 & 11 & 12 \\
\hline 1965 & 15 & 12 & 11 & 12 & 11 & 12 \\
\hline 1976 & 15 & 12 & 11 & 12 & 11 & 12 \\
\hline 1984 & 15 & 12 & 11 & 12 & 11 & 12 \\
\hline 1990 & 15 & 12 & 11 & 12 & 11 & 12 \\
\hline 2000 & 15 & 12 & 11 & 12 & 11 & 12 \\
\hline USA_GA_ATLANTA & 15 & 12 & 11 & 12 & 11 & 12 \\
\hline USA_IL_CHICAGO-OHARE & 15 & 11 & 11 & 12 & 11 & 12 \\
\hline USA_MD_BALTIMORE & 15 & 11 & 11 & 12 & 11 & 12 \\
\hline USA_CA_LOS_ANGELES & 15 & 12 & 11 & 12 & 11 & 12 \\
\hline USA_TX_HOUSTON & 15 & 12 & 11 & 12 & 11 & 12 \\
\hline
\end{tabular}


Table 8: Cooling load

\begin{tabular}{|c|c|c|c|c|c|c|}
\hline \multirow[b]{2}{*}{ Results Aggregated By: } & \multicolumn{6}{|c|}{ Cooling Load (kBtu/yr/ft2) } \\
\hline & Baseline & $\begin{array}{c}\text { Baseline } \\
\text { with } \\
\text { daylighting }\end{array}$ & $\begin{array}{c}\text { EC } \\
\text { Always } \\
\text { Off }\end{array}$ & $\begin{array}{l}\text { EC On } \\
\text { For } \\
\text { Glare }\end{array}$ & $\begin{array}{l}\text { EC On For } \\
\text { Daylighting }\end{array}$ & $\begin{array}{l}\text { EC On For } \\
\text { Glare And } \\
\text { Daylighting }\end{array}$ \\
\hline Assembly & 31 & 29 & 32 & 30 & 29 & 28 \\
\hline Education & 33 & 32 & 34 & 33 & 30 & 30 \\
\hline Food Sales & 68 & 66 & 68 & 67 & 68 & 67 \\
\hline Food Service & 66 & 62 & 64 & 62 & 63 & 62 \\
\hline Health Care & 83 & 82 & 86 & 84 & 79 & 79 \\
\hline Lodging & 42 & 39 & 45 & 42 & 34 & 34 \\
\hline Mercantile and Service & 29 & 28 & 28 & 28 & 28 & 28 \\
\hline Office & 29 & 27 & 32 & 28 & 24 & 24 \\
\hline Public Order/Safety & 26 & 25 & 26 & 25 & 24 & 24 \\
\hline Warehouse and Storage & 52 & 52 & 53 & 52 & 52 & 52 \\
\hline Other & 33 & 32 & 32 & 32 & 32 & 32 \\
\hline Single Family Detached & 25 & 24 & 26 & 24 & 26 & 25 \\
\hline Single Family Attached & 109 & 105 & 114 & 105 & 113 & 106 \\
\hline 2 to 4 Unit Buildings & 29 & 28 & 35 & 30 & 24 & 24 \\
\hline 5 or More Unit Buildings & 24 & 23 & 28 & 25 & 19 & 19 \\
\hline Mobile Homes & 36 & 35 & 39 & 34 & 34 & 34 \\
\hline 1945 & 58 & 57 & 60 & 58 & 56 & 55 \\
\hline 1955 & 55 & 54 & 57 & 55 & 53 & 52 \\
\hline 1965 & 51 & 49 & 53 & 50 & 48 & 48 \\
\hline 1976 & 41 & 40 & 43 & 40 & 39 & 39 \\
\hline 1984 & 37 & 35 & 38 & 36 & 35 & 34 \\
\hline 1990 & 35 & 33 & 36 & 34 & 32 & 32 \\
\hline 2000 & 36 & 34 & 37 & 35 & 33 & 33 \\
\hline USA_GA_ATLANTA & 46 & 45 & 49 & 46 & 45 & 44 \\
\hline USA_IL_CHICAGO-OHARE & 35 & 34 & 34 & 33 & 31 & 31 \\
\hline USA_MD_BALTIMORE & 42 & 41 & 43 & 40 & 39 & 38 \\
\hline USA_CA_LOS_ANGELES & 31 & 29 & 34 & 31 & 30 & 29 \\
\hline USA_TX_HOUSTON & 70 & 68 & 72 & 70 & 67 & 67 \\
\hline
\end{tabular}


Table 9: Heating load

\begin{tabular}{|c|c|c|c|c|c|c|}
\hline \multirow[b]{2}{*}{ Results Aggregated By: } & \multicolumn{6}{|c|}{ Heating Load (kBtu/yr/ft2) } \\
\hline & Baseline & $\begin{array}{c}\text { Baseline } \\
\text { with } \\
\text { daylighting }\end{array}$ & $\begin{array}{c}\text { EC } \\
\text { Always } \\
\text { Off }\end{array}$ & $\begin{array}{l}\text { EC On } \\
\text { For } \\
\text { Glare } \\
\end{array}$ & $\begin{array}{l}\text { EC On For } \\
\text { Daylighting }\end{array}$ & $\begin{array}{l}\text { EC On For } \\
\text { Glare And } \\
\text { Daylighting }\end{array}$ \\
\hline Assembly & 15 & 15 & 14 & 14 & 14 & 14 \\
\hline Education & 15 & 15 & 13 & 14 & 14 & 14 \\
\hline Food Sales & 14 & 14 & 13 & 14 & 13 & 14 \\
\hline Food Service & 24 & 24 & 22 & 23 & 23 & 23 \\
\hline Health Care & 63 & 63 & 59 & 62 & 59 & 59 \\
\hline Lodging & 27 & 26 & 23 & 25 & 21 & 21 \\
\hline Mercantile and Service & 20 & 21 & 20 & 20 & 20 & 20 \\
\hline Office & 10 & 10 & 7 & 9 & 8 & 8 \\
\hline Public Order/Safety & 16 & 17 & 15 & 16 & 15 & 15 \\
\hline Warehouse and Storage & 86 & 86 & 85 & 86 & 85 & 86 \\
\hline Other & 45 & 45 & 44 & 44 & 44 & 44 \\
\hline Single Family Detached & 10 & 10 & 8 & 8 & 8 & 8 \\
\hline Single Family Attached & 36 & 36 & 27 & 32 & 27 & 31 \\
\hline 2 to 4 Unit Buildings & 12 & 12 & 9 & 10 & 8 & 9 \\
\hline 5 or More Unit Buildings & 11 & 11 & 7 & 9 & 9 & 9 \\
\hline Mobile Homes & 28 & 29 & 24 & 26 & 25 & 26 \\
\hline 1945 & 40 & 40 & 37 & 39 & 37 & 38 \\
\hline 1955 & 37 & 37 & 34 & 35 & 34 & 34 \\
\hline 1965 & 32 & 32 & 29 & 30 & 29 & 30 \\
\hline 1976 & 23 & 23 & 21 & 22 & 21 & 21 \\
\hline 1984 & 20 & 20 & 17 & 19 & 18 & 18 \\
\hline 1990 & 18 & 19 & 16 & 17 & 16 & 17 \\
\hline 2000 & 19 & 19 & 16 & 18 & 17 & 17 \\
\hline USA_GA_ATLANTA & 24 & 24 & 21 & 22 & 21 & 22 \\
\hline USA_IL_CHICAGO-OHARE & 49 & 49 & 45 & 47 & 45 & 46 \\
\hline USA_MD_BALTIMORE & 38 & 38 & 35 & 37 & 35 & 35 \\
\hline USA_CA_LOS_ANGELES & 9 & 9 & 8 & 9 & 8 & 8 \\
\hline USA_TX_HOUSTON & 15 & 15 & 13 & 14 & 13 & 13 \\
\hline
\end{tabular}




\subsection{Conclusions}

The tool outlined in this report was developed in an effort to overcome the challenges hindering the use of EnergyPlus in large-scale, multi-building simulations. These challenges include the requirement for highly complex and detailed inputs, excessive simulation time, and massive quantities of output data. By reducing required inputs to a list of easily understandable variables, allowing the user to capitalize on the benefits of parallel computing, and automatically collecting the pertinent output data into a single file, the ABS methodology greatly broadens the usefulness of the EnergyPlus software.

The ABS has the potential to be put to a wide variety of uses, including simulating the overall impact of mandated changes to the built environment, parametric simulations to compare the impact of adjusting a single building attribute, as well as developing local or regional building energy consumption profiles. In general, the ABS vastly expands the control volume within which EnergyPlus can provide useful information, moving from the limitations of a single building or facility to where the scope is limited only by access to computing power and knowledge of the building stock one intends to simulate. 


\subsection{References}

National Renewable Energy Laboratory (NREL). 2008. NREL EnergyPlus Preprocessor Help Document. Golden, Colorado, September 2008.

EnergyPlus. 2010. EnergyPlus User's Manual, V5.0.0 Build 031; April 2010. Engineering Document. U.S. Department of Energy, Washington, D.C. http://www.energyplus.gov/. Last accessed July 28, 2010.

Belzer, D. 2010. An Exploratory Energy Analysis of Electrochromic Windows in Small and Medium Office Buildings - Simulated Results Using EnergyPlus. PNNL-19637. Pacific Northwest National Laboratory, Richland, Washington.

EnergyPlus Example File Generator. 2011.

http://apps1.eere.energy.gov/buildings/energyplus/cfm/inputs/index.cfm\#building_geometry/. Last accessed October 27, 2011. 


\section{APPENDIX A}

Aggregate Building Simulator Input Generator 



\section{Appendix A: Aggregate Building Simulator Input Generator}

The Aggregate Building Simulator Input Generator (ABSIG) is a tool developed through internal funding to facilitate the production of the required comma separated value (CSV) inputs for the Aggregate Building Simulator (ABS). The design intention is to allow for the production of an entire input file with a minimal knowledge of the buildings to be simulated. The users can easily specify as much detail as they wish, but as a minimum they must provide:

- Use

- Location

- Floor area

- Vintage.

From these four inputs, all of the required ABS input variables can be inferred. The inferences are derived from a number of sources, including:

- Commercial Building Energy Consumption Survey (CBECS)

- Residential Energy Consumption Survey (RECS)

- End-Use Load and Consumer Assessment Program (ELCAP)

- American Society of Heating, Refrigeration and Air Conditioning Engineers (ASHRAE) standard design and construction practices.

Inferences from these sources are arrived at in two main ways. From the survey data (CEBECS, RECS, and ELCAP), ordinary least squares dummy variable regression is used to develop the probabilities that certain building attributes exist; while the way codes and standards change over time and across locations is used to predict when the implementation of certain technologies likely occurred.

When a user enters the minimum set of information required, all of the variables required by the ABS are inferred within the excel spread sheet through a series of look up tables that populate the second sheet of the workbook. To use this auto generated input, the user must first copy the entire input sheet and paste its values alone to a new work book. The new work book can then be saved in the ABS project directory in the comma separated value format. For a detailed list of the available variables and brief descriptions of each, please see Appendix B. 


$$
\text { A-2 }
$$




\section{APPENDIX B}

Aggregate Building Simulator Input Variables, Description, Default Values 



\section{Appendix B: Aggregate Building Simulator Input Variables, Description, Default Values}

\begin{tabular}{|c|c|c|c|}
\hline Variable & Description & Default & Units \\
\hline TimeSteplnHour & $\begin{array}{c}\text { Time steps per hour used by } \\
\text { EnergyPlus }\end{array}$ & 4 & per hour \\
\hline RunStartMonth & $\begin{array}{c}\text { Begin Month For energy Plus } \\
\text { Simulation } \\
\end{array}$ & 1 & Month \\
\hline RunStartDay & $\begin{array}{c}\text { Begin day For energy Plus } \\
\text { Simulation }\end{array}$ & 1 & Day \\
\hline RunStopMonth & $\begin{array}{l}\text { End Month For energy Plus } \\
\text { Simulation } \\
\end{array}$ & 12 & Month \\
\hline RunStopDay & End day For energy Plus Simulation & 31 & Day \\
\hline MaxNumberOfWarmupDays & $\begin{array}{c}\text { Maximum days before simulation } \\
\text { that energy plus has to achieve } \\
\text { convergence }\end{array}$ & 25 & Day \\
\hline LoadConvergeTolerance & $\begin{array}{l}\text { maximum level of divergence } \\
\text { allowed in load calculations }\end{array}$ & 0.04 & \\
\hline TemperatureConvergeTolerance & $\begin{array}{l}\text { maximum level of divergence } \\
\text { allowed in temperature calculations }\end{array}$ & 0.2 & C \\
\hline UpdateShadowInterval & $\begin{array}{l}\text { how often (in days) to update } \\
\text { shadows }\end{array}$ & 30 & Day \\
\hline MaxHVACIterations & $\begin{array}{c}\text { maximum number of interrelations } \\
\text { HVAC simulation has to achieve } \\
\text { convergence }\end{array}$ & 25 & integer \\
\hline MinSystemTimeStep & $\begin{array}{l}\text { minimum timestep allowed for } \\
\text { system simulation }\end{array}$ & 5 & per hour \\
\hline EnergyPlusVersion & version of energy plus to use & 3.1 & N/A \\
\hline PrimarySpaceType & $\begin{array}{l}\text { Primary function of building to be } \\
\text { modeled }\end{array}$ & Office & N/A \\
\hline Location & Location of Building & USA_WA_SEATTLE & N/A \\
\hline ConstructsLibrary & $\begin{array}{l}\text { Library to use for default } \\
\text { constructions } \\
\end{array}$ & HPBStandardConstructions.imf & N/A \\
\hline SWinName & Name of South Window & EC_Window_Light & N/A \\
\hline SWinNumberPanes & $\begin{array}{l}\text { Number of Panes for south } \\
\text { windows }\end{array}$ & 1 & integer \\
\hline SWinGlass1Name & South window pane 1 attributes & THEORETICAL GLASS [LIGHT] & N/A \\
\hline SWinGlass1Thickness & South window pane 1 attributes & 0.05469 & Meter \\
\hline SWinGlass1SolarTrans & South window pane 1 attributes & 0.45003 & $\%$ \\
\hline SWinGlass1SolarReflectFront & South window pane 1 attributes & 0.38255 & $\%$ \\
\hline SWinGlass1SolarReflectBack & South window pane 1 attributes & 0.38255 & $\%$ \\
\hline SWinGlass1VisibleTrans & South window pane 1 attributes & 0.65 & $\%$ \\
\hline SWinGlass1VisibleReflectFront & South window pane 1 attributes & 0.13254 & $\%$ \\
\hline SWinGlass1VisibleReflectBack & South window pane 1 attributes & 0.15003 & $\%$ \\
\hline SWinGlass1IRTrans & South window pane 1 attributes & 0 & $\%$ \\
\hline SWinGlass1IREmmFront & South window pane 1 attributes & 0.84 & $\%$ \\
\hline
\end{tabular}




\begin{tabular}{|c|c|c|c|}
\hline Variable & Description & Default & Units \\
\hline SWinGlass1IREmmBack & South window pane 1 attributes & 0.84 & $\%$ \\
\hline SWinGlass1Conductivity & South window pane 1 attributes & 0.03411 & $\mathrm{~W} / \mathrm{m}-\mathrm{K}$ \\
\hline SWinGasName & South window gas attributes & 'AIR 6MM' & N/A \\
\hline SWinGasType & South window gas attributes & Air & N/A \\
\hline SWinGasThickness & South window gas attributes & 0.0063 & Meter \\
\hline SWinGlass2Name & South window pane 2 attributes & Theoretical Glass [117] & N/A \\
\hline SWinGlass2Thickness & South window pane 2 attributes & 0.003 & Meter \\
\hline SWinGlass2SolarTrans & South window pane 2 attributes & 0.39333 & $\%$ \\
\hline SWinGlass2SolarReflectFront & South window pane 2 attributes & 0.55667 & $\%$ \\
\hline SWinGlass2SolarReflectBack & South window pane 2 attributes & 0.55667 & $\%$ \\
\hline SWinGlass2VisibleTrans & South window pane 2 attributes & 0.507883 & $\%$ \\
\hline SWinGlass2VisibleReflectFront & South window pane 2 attributes & 0.442117 & $\%$ \\
\hline SWinGlass2VisibleReflectBack & South window pane 2 attributes & 0.442117 & $\%$ \\
\hline SWinGlass2IRTrans & South window pane 2 attributes & 0 & $\%$ \\
\hline SWinGlass2IREmmFront & South window pane 2 attributes & 0.84 & $\%$ \\
\hline SWinGlass2IREmmBack & South window pane 2 attributes & 0.84 & $\%$ \\
\hline SWinGlass2Conductivity & South window pane 2 attributes & 0.9 & $\mathrm{~W} / \mathrm{m}-\mathrm{K}$ \\
\hline SWinGlass3Name & South window pane 3 attributes & Theoretical Glass [117] & N/A \\
\hline SWinGlass3Thickness & South window pane 3 attributes & 0.003 & Meter \\
\hline SWinGlass3SolarTrans & South window pane 3 attributes & 0.39333 & $\%$ \\
\hline SWinGlass3SolarReflectFront & South window pane 3 attributes & 0.55667 & $\%$ \\
\hline SWinGlass3SolarReflectBack & South window pane 3 attributes & 0.55667 & $\%$ \\
\hline SWinGlass3VisibleTrans & South window pane 3 attributes & 0.507883 & $\%$ \\
\hline SWinGlass3VisibleReflectFront & South window pane 3 attributes & 0.442117 & $\%$ \\
\hline SWinGlass3VisibleReflectBack & South window pane 3 attributes & 0.442117 & $\%$ \\
\hline SWinGlass3IRTrans & South window pane 3 attributes & 0 & $\%$ \\
\hline SWinGlass3IREmmFront & South window pane 3 attributes & 0.84 & $\%$ \\
\hline SWinGlass3IREmmBack & South window pane 3 attributes & 0.84 & $\%$ \\
\hline SWinGlass3Conductivity & South window pane 3 attributes & 0.9 & $\mathrm{~W} / \mathrm{m}-\mathrm{K}$ \\
\hline SWinGlass4Name & South window pane 4 attributes & Theoretical Glass [117] & N/A \\
\hline SWinGlass4Thickness & South window pane 4 attributes & 0.003 & Meter \\
\hline SWinGlass4SolarTrans & South window pane 4 attributes & 0.39333 & $\%$ \\
\hline SWinGlass4SolarReflectFront & South window pane 4 attributes & 0.55667 & $\%$ \\
\hline SWinGlass4SolarReflectBack & South window pane 4 attributes & 0.55667 & $\%$ \\
\hline SWinGlass4VisibleTrans & South window pane 4 attributes & 0.507883 & $\%$ \\
\hline SWinGlass4VisibleReflectFront & South window pane 4 attributes & 0.442117 & $\%$ \\
\hline SWinGlass4VisibleReflectBack & South window pane 4 attributes & 0.442117 & $\%$ \\
\hline SWinGlass4IRTrans & South window pane 4 attributes & 0 & $\%$ \\
\hline SWinGlass4IREmmFront & South window pane 4 attributes & 0.84 & $\%$ \\
\hline
\end{tabular}

\section{B-2}




\begin{tabular}{|c|c|c|c|}
\hline Variable & Description & Default & Units \\
\hline SWinGlass4IREmmBack & South window pane 4 attributes & 0.84 & $\%$ \\
\hline SWinGlass4Conductivity & South window pane 4 attributes & 0.9 & $\mathrm{~W} / \mathrm{m}-\mathrm{K}$ \\
\hline EWinName & Name of East Window & EC_Window_Light & N/A \\
\hline EWinNumberPanes & Number of Panes for East windows & 1 & integer \\
\hline EWinGlass1Name & East window pane 1 attributes & THEORETICAL GLASS [LIGHT] & N/A \\
\hline EWinGlass1Thickness & East window pane 1 attributes & 0.05469 & Meter \\
\hline EWinGlass1SolarTrans & East window pane 1 attributes & 0.45003 & $\%$ \\
\hline EWinGlass1SolarReflectFront & East window pane 1 attributes & 0.38255 & $\%$ \\
\hline EWinGlass1SolarReflectBack & East window pane 1 attributes & 0.38255 & $\%$ \\
\hline EWinGlass1VisibleTrans & East window pane 1 attributes & 0.65 & $\%$ \\
\hline EWinGlass1VisibleReflectFront & East window pane 1 attributes & 0.13254 & $\%$ \\
\hline EWinGlass1VisibleReflectBack & East window pane 1 attributes & 0.15003 & $\%$ \\
\hline EWinGlass1IRTrans & East window pane 1 attributes & 0 & $\%$ \\
\hline EWinGlass1IREmmFront & East window pane 1 attributes & 0.84 & $\%$ \\
\hline EWinGlass1IREmmBack & East window pane 1 attributes & 0.84 & $\%$ \\
\hline EWinGlass1Conductivity & East window pane 1 attributes & 0.03411 & $\mathrm{~W} / \mathrm{m}-\mathrm{K}$ \\
\hline EWinGasName & East window gas attributes & 'AIR 6MM' & N/A \\
\hline EWinGasType & East window gas attributes & Air & N/A \\
\hline EWinGasThickness & East window gas attributes & 0.0063 & Meter \\
\hline EWinGlass2Name & East window pane 2 attributes & Theoretical Glass [117] & N/A \\
\hline EWinGlass2Thickness & East window pane 2 attributes & 0.003 & Meter \\
\hline EWinGlass2SolarTrans & East window pane 2 attributes & 0.39333 & $\%$ \\
\hline EWinGlass2SolarReflectFront & East window pane 2 attributes & 0.55667 & $\%$ \\
\hline EWinGlass2SolarReflectBack & East window pane 2 attributes & 0.55667 & $\%$ \\
\hline EWinGlass2VisibleTrans & East window pane 2 attributes & 0.507883 & $\%$ \\
\hline EWinGlass2VisibleReflectFront & East window pane 2 attributes & 0.442117 & $\%$ \\
\hline EWinGlass2VisibleReflectBack & East window pane 2 attributes & 0.442117 & $\%$ \\
\hline EWinGlass2IRTrans & East window pane 2 attributes & 0 & $\%$ \\
\hline EWinGlass2IREmmFront & East window pane 2 attributes & 0.84 & $\%$ \\
\hline EWinGlass2IREmmBack & East window pane 2 attributes & 0.84 & $\%$ \\
\hline EWinGlass2Conductivity & East window pane 2 attributes & 0.9 & $\mathrm{~W} / \mathrm{m}-\mathrm{K}$ \\
\hline EWinGlass3Name & East window pane 3 attributes & Theoretical Glass [117] & N/A \\
\hline EWinGlass3Thickness & East window pane 3 attributes & 0.003 & Meter \\
\hline EWinGlass3SolarTrans & East window pane 3 attributes & 0.39333 & $\%$ \\
\hline EWinGlass3SolarReflectFront & East window pane 3 attributes & 0.55667 & $\%$ \\
\hline EWinGlass3SolarReflectBack & East window pane 3 attributes & 0.55667 & $\%$ \\
\hline EWinGlass3VisibleTrans & East window pane 3 attributes & 0.507883 & $\%$ \\
\hline EWinGlass3VisibleReflectFront & East window pane 3 attributes & 0.442117 & $\%$ \\
\hline EWinGlass3VisibleReflectBack & East window pane 3 attributes & 0.442117 & $\%$ \\
\hline
\end{tabular}

\section{B-3}




\begin{tabular}{|c|c|c|c|}
\hline Variable & Description & Default & Units \\
\hline EWinGlass3IRTrans & East window pane 3 attributes & 0 & $\%$ \\
\hline EWinGlass3IREmmFront & East window pane 3 attributes & 0.84 & $\%$ \\
\hline EWinGlass3IREmmBack & East window pane 3 attributes & 0.84 & $\%$ \\
\hline EWinGlass3Conductivity & East window pane 3 attributes & 0.9 & $\mathrm{~W} / \mathrm{m}-\mathrm{K}$ \\
\hline EWinGlass4Name & East window pane 4 attributes & Theoretical Glass [117] & N/A \\
\hline EWinGlass4Thickness & East window pane 4 attributes & 0.003 & Meter \\
\hline EWinGlass4SolarTrans & East window pane 4 attributes & 0.39333 & $\%$ \\
\hline EWinGlass4SolarReflectFront & East window pane 4 attributes & 0.55667 & $\%$ \\
\hline EWinGlass4SolarReflectBack & East window pane 4 attributes & 0.55667 & $\%$ \\
\hline EWinGlass4VisibleTrans & East window pane 4 attributes & 0.507883 & $\%$ \\
\hline EWinGlass4VisibleReflectFront & East window pane 4 attributes & 0.442117 & $\%$ \\
\hline EWinGlass4VisibleReflectBack & East window pane 4 attributes & 0.442117 & $\%$ \\
\hline EWinGlass4IRTrans & East window pane 4 attributes & 0 & $\%$ \\
\hline EWinGlass4IREmmFront & East window pane 4 attributes & 0.84 & $\%$ \\
\hline EWinGlass4IREmmBack & East window pane 4 attributes & 0.84 & $\%$ \\
\hline EWinGlass4Conductivity & East window pane 4 attributes & 0.9 & $\mathrm{~W} / \mathrm{m}-\mathrm{K}$ \\
\hline NWinName & Name of North Window & EC_Window_Light & N/A \\
\hline NWinNumberPanes & $\begin{array}{l}\text { Number of Panes for North } \\
\text { windows }\end{array}$ & 1 & integer \\
\hline NWinGlass1Name & North window pane 1 attributes & THEORETICAL GLASS [LIGHT] & N/A \\
\hline NWinGlass1Thickness & North window pane 1 attributes & 0.05469 & Meter \\
\hline NWinGlass1SolarTrans & North window pane 1 attributes & 0.45003 & $\%$ \\
\hline NWinGlass1SolarReflectFront & North window pane 1 attributes & 0.38255 & $\%$ \\
\hline NWinGlass1SolarReflectBack & North window pane 1 attributes & 0.38255 & $\%$ \\
\hline NWinGlass1VisibleTrans & North window pane 1 attributes & 0.65 & $\%$ \\
\hline NWinGlass1VisibleReflectFront & North window pane 1 attributes & 0.13254 & $\%$ \\
\hline NWinGlass1VisibleReflectBack & North window pane 1 attributes & 0.15003 & $\%$ \\
\hline NWinGlass1IRTrans & North window pane 1 attributes & 0 & $\%$ \\
\hline NWinGlass1IREmmFront & North window pane 1 attributes & 0.84 & $\%$ \\
\hline NWinGlass1IREmmBack & North window pane 1 attributes & 0.84 & $\%$ \\
\hline NWinGlass1Conductivity & North window pane 1 attributes & 0.03411 & $\mathrm{~W} / \mathrm{m}-\mathrm{K}$ \\
\hline NWinGasName & North window gas attributes & 'AIR 6MM' & N/A \\
\hline NWinGasType & North window gas attributes & Air & N/A \\
\hline NWinGasThickness & North window gas attributes & 0.0063 & Meter \\
\hline NWinGlass2Name & North window pane 2 attributes & Theoretical Glass [117] & N/A \\
\hline NWinGlass2Thickness & North window pane 2 attributes & 0.003 & Meter \\
\hline NWinGlass2SolarTrans & North window pane 2 attributes & 0.39333 & $\%$ \\
\hline NWinGlass2SolarReflectFront & North window pane 2 attributes & 0.55667 & $\%$ \\
\hline NWinGlass2SolarReflectBack & North window pane 2 attributes & 0.55667 & $\%$ \\
\hline NWinGlass2VisibleTrans & North window pane 2 attributes & 0.507883 & $\%$ \\
\hline
\end{tabular}




\begin{tabular}{|c|c|c|c|}
\hline Variable & Description & Default & Units \\
\hline NWinGlass2VisibleReflectFront & North window pane 2 attributes & 0.442117 & $\%$ \\
\hline NWinGlass2VisibleReflectBack & North window pane 2 attributes & 0.442117 & $\%$ \\
\hline NWinGlass2IRTrans & North window pane 2 attributes & 0 & $\%$ \\
\hline NWinGlass2IREmmFront & North window pane 2 attributes & 0.84 & $\%$ \\
\hline NWinGlass2IREmmBack & North window pane 2 attributes & 0.84 & $\%$ \\
\hline NWinGlass2Conductivity & North window pane 2 attributes & 0.9 & $\mathrm{~W} / \mathrm{m}-\mathrm{K}$ \\
\hline NWinGlass3Name & North window pane 3 attributes & Theoretical Glass [117] & N/A \\
\hline NWinGlass3Thickness & North window pane 3 attributes & 0.003 & Meter \\
\hline NWinGlass3SolarTrans & North window pane 3 attributes & 0.39333 & $\%$ \\
\hline NWinGlass3SolarReflectFront & North window pane 3 attributes & 0.55667 & $\%$ \\
\hline NWinGlass3SolarReflectBack & North window pane 3 attributes & 0.55667 & $\%$ \\
\hline NWinGlass3VisibleTrans & North window pane 3 attributes & 0.507883 & $\%$ \\
\hline NWinGlass3VisibleReflectFront & North window pane 3 attributes & 0.442117 & $\%$ \\
\hline NWinGlass3VisibleReflectBack & North window pane 3 attributes & 0.442117 & $\%$ \\
\hline NWinGlass3IRTrans & North window pane 3 attributes & 0 & $\%$ \\
\hline NWinGlass3IREmmFront & North window pane 3 attributes & 0.84 & $\%$ \\
\hline NWinGlass3IREmmBack & North window pane 3 attributes & 0.84 & $\%$ \\
\hline NWinGlass3Conductivity & North window pane 3 attributes & 0.9 & $\mathrm{~W} / \mathrm{m}-\mathrm{K}$ \\
\hline NWinGlass4Name & North window pane 4 attributes & Theoretical Glass [117] & N/A \\
\hline NWinGlass4Thickness & North window pane 4 attributes & 0.003 & Meter \\
\hline NWinGlass4SolarTrans & North window pane 4 attributes & 0.39333 & $\%$ \\
\hline NWinGlass4SolarReflectFront & North window pane 4 attributes & 0.55667 & $\%$ \\
\hline NWinGlass4SolarReflectBack & North window pane 4 attributes & 0.55667 & $\%$ \\
\hline NWinGlass4VisibleTrans & North window pane 4 attributes & 0.507883 & $\%$ \\
\hline NWinGlass4VisibleReflectFront & North window pane 4 attributes & 0.442117 & $\%$ \\
\hline NWinGlass4VisibleReflectBack & North window pane 4 attributes & 0.442117 & $\%$ \\
\hline NWinGlass4IRTrans & North window pane 4 attributes & 0 & $\%$ \\
\hline NWinGlass4IREmmFront & North window pane 4 attributes & 0.84 & $\%$ \\
\hline NWinGlass4IREmmBack & North window pane 4 attributes & 0.84 & $\%$ \\
\hline NWinGlass4Conductivity & North window pane 4 attributes & 0.9 & $\mathrm{~W} / \mathrm{m}-\mathrm{K}$ \\
\hline WWinName & Name of West Window & EC_Window_Light & N/A \\
\hline WWinNumberPanes & Number of Panes for West windows & 1 & integer \\
\hline WWinGlass1Name & West window pane 1 attributes & THEORETICAL GLASS [LIGHT] & N/A \\
\hline WWinGlass1Thickness & West window pane 1 attributes & 0.05469 & Meter \\
\hline WWinGlass1SolarTrans & West window pane 1 attributes & 0.45003 & $\%$ \\
\hline WWinGlass1SolarReflectFront & West window pane 1 attributes & 0.38255 & $\%$ \\
\hline WWinGlass1SolarReflectBack & West window pane 1 attributes & 0.38255 & $\%$ \\
\hline WWinGlass1VisibleTrans & West window pane 1 attributes & 0.65 & $\%$ \\
\hline WWinGlass1VisibleReflectFront & West window pane 1 attributes & 0.13254 & $\%$ \\
\hline
\end{tabular}

\section{B-5}




\begin{tabular}{|c|c|c|c|}
\hline Variable & Description & Default & Units \\
\hline WWinGlass1VisibleReflectBack & West window pane 1 attributes & 0.15003 & $\%$ \\
\hline WWinGlass1IRTrans & West window pane 1 attributes & 0 & $\%$ \\
\hline WWinGlass1IREmmFront & West window pane 1 attributes & 0.84 & $\%$ \\
\hline WWinGlass1IREmmBack & West window pane 1 attributes & 0.84 & $\%$ \\
\hline WWinGlass1Conductivity & West window pane 1 attributes & 0.03411 & $\mathrm{~W} / \mathrm{m}-\mathrm{K}$ \\
\hline WWinGasName & West window gas attributes & 'AIR 6MM' & $\mathrm{N} / \mathrm{A}$ \\
\hline WWinGasType & West window gas attributes & Air & N/A \\
\hline WWinGasThickness & West window gas attributes & 0.0063 & Meter \\
\hline WWinGlass2 Name & West window pane 2 attributes & Theoretical Glass [117] & $\mathrm{N} / \mathrm{A}$ \\
\hline WWinGlass2Thickness & West window pane 2 attributes & 0.003 & Meter \\
\hline WWinGlass2SolarTrans & West window pane 2 attributes & 0.39333 & $\%$ \\
\hline WWinGlass2SolarReflectFront & West window pane 2 attributes & 0.55667 & $\%$ \\
\hline WWinGlass2SolarReflectBack & West window pane 2 attributes & 0.55667 & $\%$ \\
\hline WWinGlass2VisibleTrans & West window pane 2 attributes & 0.507883 & $\%$ \\
\hline WWinGlass2VisibleReflectFront & West window pane 2 attributes & 0.442117 & $\%$ \\
\hline WWinGlass2VisibleReflectBack & West window pane 2 attributes & 0.442117 & $\%$ \\
\hline WWinGlass2IRTrans & West window pane 2 attributes & 0 & $\%$ \\
\hline WWinGlass2IREmmFront & West window pane 2 attributes & 0.84 & $\%$ \\
\hline WWinGlass2IREmmBack & West window pane 2 attributes & 0.84 & $\%$ \\
\hline WWinGlass2Conductivity & West window pane 2 attributes & 0.9 & $\mathrm{~W} / \mathrm{m}-\mathrm{K}$ \\
\hline WWinGlass3Name & West window pane 3 attributes & Theoretical Glass [117] & $\mathrm{N} / \mathrm{A}$ \\
\hline WWinGlass3Thickness & West window pane 3 attributes & 0.003 & Meter \\
\hline WWinGlass3SolarTrans & West window pane 3 attributes & 0.39333 & $\%$ \\
\hline WWinGlass3SolarReflectFront & West window pane 3 attributes & 0.55667 & $\%$ \\
\hline WWinGlass3SolarReflectBack & West window pane 3 attributes & 0.55667 & $\%$ \\
\hline WWinGlass3VisibleTrans & West window pane 3 attributes & 0.507883 & $\%$ \\
\hline WWinGlass3VisibleReflectFront & West window pane 3 attributes & 0.442117 & $\%$ \\
\hline WWinGlass3VisibleReflectBack & West window pane 3 attributes & 0.442117 & $\%$ \\
\hline WWinGlass3IRTrans & West window pane 3 attributes & 0 & $\%$ \\
\hline WWinGlass3IREmmFront & West window pane 3 attributes & 0.84 & $\%$ \\
\hline WWinGlass3IREmmBack & West window pane 3 attributes & 0.84 & $\%$ \\
\hline WWinGlass3Conductivity & West window pane 3 attributes & 0.9 & $\mathrm{~W} / \mathrm{m}-\mathrm{K}$ \\
\hline WWinGlass4Name & West window pane 4 attributes & Theoretical Glass [117] & N/A \\
\hline WWinGlass4Thickness & West window pane 4 attributes & 0.003 & Meter \\
\hline WWinGlass4SolarTrans & West window pane 4 attributes & 0.39333 & $\%$ \\
\hline WWinGlass4SolarReflectFront & West window pane 4 attributes & 0.55667 & $\%$ \\
\hline WWinGlass4SolarReflectBack & West window pane 4 attributes & 0.55667 & $\%$ \\
\hline WWinGlass4VisibleTrans & West window pane 4 attributes & 0.507883 & $\%$ \\
\hline WWinGlass4VisibleReflectFront & West window pane 4 attributes & 0.442117 & $\%$ \\
\hline
\end{tabular}

\section{B-6}




\begin{tabular}{|c|c|c|c|}
\hline Variable & Description & Default & Units \\
\hline WWinGlass4VisibleReflectBack & West window pane 4 attributes & 0.442117 & $\%$ \\
\hline WWinGlass4IRTrans & West window pane 4 attributes & 0 & $\%$ \\
\hline WWinGlass4IREmmFront & West window pane 4 attributes & 0.84 & $\%$ \\
\hline WWinGlass4IREmmBack & West window pane 4 attributes & 0.84 & $\%$ \\
\hline WWinGlass4Conductivity & West window pane 4 attributes & 0.9 & $\mathrm{~W} / \mathrm{m}-\mathrm{K}$ \\
\hline SkyLiName & Name of Sky Light & $\begin{array}{c}\text { Dbl LoE (e2=.2) Clr } 6 \mathrm{~mm} / 6 \mathrm{~mm} \\
\text { Air }\end{array}$ & N/A \\
\hline SkyLiNumberPanes & Number of Panes for Sky Light & 2 & integer \\
\hline SkyLiType & Describes Installation Type & 'All Skylights without Curb' & N/A \\
\hline SkyLilnstallConfig & Describes distribution of skylights & 'All Top Zones' & N/A \\
\hline SkyLiAreaFrac & $\begin{array}{l}\text { Roof Area percentage covered by } \\
\text { skylights }\end{array}$ & 0 & $\%$ \\
\hline SkyLiGlass1Name & Sky Light pane 1 attributes & 'PYR B CLEAR 6MM' & N/A \\
\hline SkyLiGlass1Thickness & Sky Light pane 1 attributes & 0.006 & Meter \\
\hline SkyLiGlass1SolarTrans & Sky Light pane 1 attributes & 0.68 & $\%$ \\
\hline SkyLiGlass1SolarReflectFront & Sky Light pane 1 attributes & 0.09 & $\%$ \\
\hline SkyLiGlass1SolarReflectBack & Sky Light pane 1 attributes & 0.1 & $\%$ \\
\hline SkyLiGlass1VisibleTrans & Sky Light pane 1 attributes & 0.81 & $\%$ \\
\hline SkyLiGlass1VisibleReflectFront & Sky Light pane 1 attributes & 0.11 & $\%$ \\
\hline SkyLiGlass1VisibleReflectBack & Sky Light pane 1 attributes & 0.12 & $\%$ \\
\hline SkyLiGlass1IRTrans & Sky Light pane 1 attributes & 0 & $\%$ \\
\hline SkyLiGlass1IREmmFront & Sky Light pane 1 attributes & 0.84 & $\%$ \\
\hline SkyLiGlass1IREmmBack & Sky Light pane 1 attributes & 0.2 & $\%$ \\
\hline SkyLiGlass1Conductivity & Sky Light pane 1 attributes & 0.9 & $\mathrm{~W} / \mathrm{m}-\mathrm{K}$ \\
\hline SkyLiGasName & Sky Light gas attributes & 'AIR 6MM' & N/A \\
\hline SkyLiGasType & Sky Light gas attributes & 'Air' & N/A \\
\hline SkyLiGasThickness & Sky Light gas attributes & 0.0063 & Meter \\
\hline SkyLiGlass2Name & Sky Light pane 2 attributes & 'CLEAR 6MM' & N/A \\
\hline SkyLiGlass2Thickness & Sky Light pane 2 attributes & 0.006 & Meter \\
\hline SkyLiGlass2SolarTrans & Sky Light pane 2 attributes & 0.775 & $\%$ \\
\hline SkyLiGlass2SolarReflectFront & Sky Light pane 2 attributes & 0.071 & $\%$ \\
\hline SkyLiGlass2SolarReflectBack & Sky Light pane 2 attributes & 0.071 & $\%$ \\
\hline SkyLiGlass2VisibleTrans & Sky Light pane 2 attributes & 0.881 & $\%$ \\
\hline SkyLiGlass2VisibleReflectFront & Sky Light pane 2 attributes & 0.08 & $\%$ \\
\hline SkyLiGlass2VisibleReflectBack & Sky Light pane 2 attributes & 0.08 & $\%$ \\
\hline SkyLiGlass2IRTrans & Sky Light pane 2 attributes & 0 & $\%$ \\
\hline SkyLiGlass2IREmmFront & Sky Light pane 2 attributes & 0.84 & $\%$ \\
\hline SkyLiGlass2IREmmBack & Sky Light pane 2 attributes & 0.84 & $\%$ \\
\hline SkyLiGlass2Conductivity & Sky Light pane 2 attributes & 0.9 & $\mathrm{~W} / \mathrm{m}-\mathrm{K}$ \\
\hline RoofNumLayers & $\begin{array}{c}\text { Number of layers in roof } \\
\text { construction }\end{array}$ & 3 & integer \\
\hline
\end{tabular}




\begin{tabular}{|c|c|c|c|}
\hline Variable & Description & Default & Units \\
\hline RoofDesc & Description of roof construction & IEAD_R-20 ci_Roof & N/A \\
\hline RoofType & Type of Roof & Insulation Entirely Above Deck & N/A \\
\hline RoofLayer1Name & Roof Layer 1 attributes & Roof Membrane & N/A \\
\hline RoofLayer1Roughness & Roof Layer 1 attributes & VeryRough & N/A \\
\hline RoofLayer1Thickness & Roof Layer 1 attributes & 0.0095 & Meter \\
\hline RoofLayer1Conductivity & Roof Layer 1 attributes & 0.16 & $\mathrm{~W} / \mathrm{m}-\mathrm{K}$ \\
\hline RoofLayer1Density & Roof Layer 1 attributes & 1121.29 & $\mathrm{~kg} / \mathrm{m} 3$ \\
\hline RoofLayer1SpecificHeat & Roof Layer 1 attributes & 1460 & $J($ kg-K) \\
\hline RoofLayer1AbsorThermal & Roof Layer 1 attributes & 0.9 & $\%$ \\
\hline RoofLayer1AbsorSolar & Roof Layer 1 attributes & 0.7 & $\%$ \\
\hline RoofLayer1AbsorVis & Roof Layer 1 attributes & 0.7 & $\%$ \\
\hline RoofLayer2Name & Roof Layer 2 attributes & Roof Insulation [13] & N/A \\
\hline RoofLayer2Roughness & Roof Layer 2 attributes & MediumRough & N/A \\
\hline RoofLayer2Thickness & Roof Layer 2 attributes & 0.167335 & Meter \\
\hline RoofLayer2Conductivity & Roof Layer 2 attributes & 0.049 & $\mathrm{~W} / \mathrm{m}-\mathrm{K}$ \\
\hline RoofLayer2Density & Roof Layer 2 attributes & 265 & $\mathrm{~kg} / \mathrm{m} 3$ \\
\hline RoofLayer2SpecificHeat & Roof Layer 2 attributes & 836.8 & $J(k g-K)$ \\
\hline RoofLayer2AbsorThermal & Roof Layer 2 attributes & 0.9 & $\%$ \\
\hline RoofLayer2AbsorSolar & Roof Layer 2 attributes & 0.7 & $\%$ \\
\hline RoofLayer2AbsorVis & Roof Layer 2 attributes & 0.7 & $\%$ \\
\hline RoofLayer3Name & Roof Layer 3 attributes & Metal Decking & N/A \\
\hline RoofLayer3Roughness & Roof Layer 3 attributes & MediumSmooth & N/A \\
\hline RoofLayer3Thickness & Roof Layer 3 attributes & 0.001524 & Meter \\
\hline RoofLayer3Conductivity & Roof Layer 3 attributes & 45.006 & $\mathrm{~W} / \mathrm{m}-\mathrm{K}$ \\
\hline RoofLayer3Density & Roof Layer 3 attributes & 7680 & $\mathrm{~kg} / \mathrm{m} 3$ \\
\hline RoofLayer3SpecificHeat & Roof Layer 3 attributes & 418.4 & $\mathrm{~J}(\mathrm{~kg}-\mathrm{K})$ \\
\hline RoofLayer3AbsorThermal & Roof Layer 3 attributes & 0.9 & $\%$ \\
\hline RoofLayer3AbsorSolar & Roof Layer 3 attributes & 0.7 & $\%$ \\
\hline RoofLayer3AbsorVis & Roof Layer 3 attributes & 0.3 & $\%$ \\
\hline IntWallDesc & Interior Wall Description & Standard_Int-Wall & N/A \\
\hline IntWallType & Interior Wall Type & Standard & N/A \\
\hline IntWallLayer1Name & Interior Wall Layer 1 Attributes & GP01 1/2 GYPSUM & N/A \\
\hline IntWallLayer1Roughness & Interior Wall Layer 1 Attributes & Smooth & $\mathrm{N} / \mathrm{A}$ \\
\hline IntWallLayer1Thickness & Interior Wall Layer 1 Attributes & 0.01271 & Meter \\
\hline IntWallLayer1Conductivity & Interior Wall Layer 1 Attributes & 0.16 & $\mathrm{~W} / \mathrm{m}-\mathrm{K}$ \\
\hline IntWallLayer1Density & Interior Wall Layer 1 Attributes & 800 & $\mathrm{~kg} / \mathrm{m} 3$ \\
\hline IntWallLayer1SpecificHeat & Interior Wall Layer 1 Attributes & 1090 & $J(k g-K)$ \\
\hline IntWallLayer1AbsorThermal & Interior Wall Layer 1 Attributes & 0.9 & $\%$ \\
\hline IntWallLayer1AbsorSolar & Interior Wall Layer 1 Attributes & 0.7 & $\%$ \\
\hline
\end{tabular}




\begin{tabular}{|c|c|c|c|}
\hline Variable & Description & Default & Units \\
\hline IntWallLayer1AbsorVis & Interior Wall Layer 1 Attributes & 0.5 & $\%$ \\
\hline IntWallLayer2Name & Interior Wall Layer 2 Attributes & GP01 1/2 GYPSUM & N/A \\
\hline IntWallLayer2Roughness & Interior Wall Layer 2 Attributes & Smooth & N/A \\
\hline IntWallLayer2Thickness & Interior Wall Layer 2 Attributes & 0.01271 & Meter \\
\hline IntWallLayer2Conductivity & Interior Wall Layer 2 Attributes & 0.16 & $\mathrm{~W} / \mathrm{m}-\mathrm{K}$ \\
\hline IntWallLayer2Density & Interior Wall Layer 2 Attributes & 800 & $\mathrm{~kg} / \mathrm{m} 3$ \\
\hline IntWallLayer2SpecificHeat & Interior Wall Layer 2 Attributes & 1090 & $J(k g-K)$ \\
\hline IntWallLayer2AbsorThermal & Interior Wall Layer 2 Attributes & 0.9 & $\%$ \\
\hline IntWallLayer2AbsorSolar & Interior Wall Layer 2 Attributes & 0.7 & $\%$ \\
\hline IntWallLayer2AbsorVis & Interior Wall Layer 2 Attributes & 0.5 & $\%$ \\
\hline ExtWallNumLayers & Exterior Walls Number of Layers & 3 & \\
\hline ExtWallDesc & Exterior Walls Description & $\begin{array}{c}\text { Steel-Framed_R-13 + R-7.5 } \\
\text { ci_Ext-wall }\end{array}$ & N/A \\
\hline ExtWallType & Exterior Walls Type & Steel-Framed & N/A \\
\hline ExtWallLayer1Name & Exterior Wall Layer 1 Attributes & $\begin{array}{c}\text { Composite } 2 \times 4 \text { Steel Stud R11 } \\
\# 3\end{array}$ & N/A \\
\hline ExtWallLayer1Roughness & Exterior Wall Layer 1 Attributes & Smooth & N/A \\
\hline ExtWallLayer1Thickness & Exterior Wall Layer 1 Attributes & 0.025 & Meter \\
\hline ExtWallLayer1Conductivity & Exterior Wall Layer 1 Attributes & 0.452 & $\mathrm{~W} / \mathrm{m}-\mathrm{K}$ \\
\hline ExtWallLayer1Density & Exterior Wall Layer 1 Attributes & 413.782 & $\mathrm{~kg} / \mathrm{m} 3$ \\
\hline ExtWallLayer1SpecificHeat & Exterior Wall Layer 1 Attributes & 1048 & $\mathrm{~J}(\mathrm{~kg}-\mathrm{K})$ \\
\hline ExtWallLayer1AbsorThermal & Exterior Wall Layer 1 Attributes & 0.9 & $\%$ \\
\hline ExtWallLayer1AbsorSolar & Exterior Wall Layer 1 Attributes & 0.7 & $\%$ \\
\hline ExtWallLayer1AbsorVis & Exterior Wall Layer 1 Attributes & 0.7 & $\%$ \\
\hline ExtWallLayer2Name & Exterior Wall Layer 2 Attributes & $\begin{array}{c}\text { Composite } 2 \times 4 \text { Steel Stud R11 } \\
\# 2\end{array}$ & N/A \\
\hline ExtWallLayer2Roughness & Exterior Wall Layer 2 Attributes & Smooth & N/A \\
\hline ExtWallLayer2Thickness & Exterior Wall Layer 2 Attributes & 0.089 & Meter \\
\hline ExtWallLayer2Conductivity & Exterior Wall Layer 2 Attributes & 0.06 & $\mathrm{~W} / \mathrm{m}-\mathrm{K}$ \\
\hline ExtWallLayer2Density & Exterior Wall Layer 2 Attributes & 118.223 & $\mathrm{~kg} / \mathrm{m} 3$ \\
\hline ExtWallLayer2SpecificHeat & Exterior Wall Layer 2 Attributes & 1048 & $\mathrm{~J}(\mathrm{~kg}-\mathrm{K})$ \\
\hline ExtWallLayer2AbsorThermal & Exterior Wall Layer 2 Attributes & 0.9 & $\%$ \\
\hline ExtWallLayer2AbsorSolar & Exterior Wall Layer 2 Attributes & 0.7 & $\%$ \\
\hline ExtWallLayer2AbsorVis & Exterior Wall Layer 2 Attributes & 0.7 & $\%$ \\
\hline ExtWallLayer3Name & Exterior Wall Layer 3 Attributes & $\begin{array}{c}\text { Composite 2x4 Steel Stud R11 } \\
\# 1\end{array}$ & N/A \\
\hline ExtWallLayer3Roughness & Exterior Wall Layer 3 Attributes & Smooth & N/A \\
\hline ExtWallLayer3Thickness & Exterior Wall Layer 3 Attributes & 0.013 & Meter \\
\hline ExtWallLayer3Conductivity & Exterior Wall Layer 3 Attributes & 0.72 & $\mathrm{~W} / \mathrm{m}-\mathrm{K}$ \\
\hline ExtWallLayer3Density & Exterior Wall Layer 3 Attributes & 640 & $\mathrm{~kg} / \mathrm{m} 3$ \\
\hline ExtWallLayer3SpecificHeat & Exterior Wall Layer 3 Attributes & 1048 & $J($ kg-K) \\
\hline
\end{tabular}




\begin{tabular}{|c|c|c|c|}
\hline Variable & Description & Default & Units \\
\hline ExtWallLayer3AbsorThermal & Exterior Wall Layer 3 Attributes & 0.9 & $\%$ \\
\hline ExtWallLayer3AbsorSolar & Exterior Wall Layer 3 Attributes & 0.7 & $\%$ \\
\hline ExtWallLayer3AbsorVis & Exterior Wall Layer 3 Attributes & 0.7 & $\%$ \\
\hline ExtSlabNumLayers & Exterior Slab Number of Layers & 2 & integer \\
\hline ExtSlabConstDesc & Exterior Slab Description & $\begin{array}{l}\text { Unheated - 8in Slab with } \\
\text { Carpet_Ext-slab }\end{array}$ & N/A \\
\hline ExtSlabConstrutionType & Exterior Slab Type & $\begin{array}{c}\text { Unheated - 8in Slab with } \\
\text { Carpet }\end{array}$ & N/A \\
\hline ExtSlabLayer1Name & Exterior Slab Layer 1 Attributes & MAT-CCO5 8 HW CONCRETE & N/A \\
\hline ExtSlabLayer1Roughness & Exterior Slab Layer 1 Attributes & Rough & N/A \\
\hline ExtSlabLayer1Thickness & Exterior Slab Layer 1 Attributes & 0.20321 & Meter \\
\hline ExtSlabLayer1Conductivity & Exterior Slab Layer 1 Attributes & 1.311 & $\mathrm{~W} / \mathrm{m}-\mathrm{K}$ \\
\hline ExtSlabLayer1Density & Exterior Slab Layer 1 Attributes & 2240 & $\mathrm{~kg} / \mathrm{m} 3$ \\
\hline ExtSlabLayer1SpecificHeat & Exterior Slab Layer 1 Attributes & 836.8 & $\mathrm{~J}(\mathrm{~kg}-\mathrm{K})$ \\
\hline ExtSlabLayer1AbsorThermal & Exterior Slab Layer 1 Attributes & 0.9 & $\%$ \\
\hline ExtSlabLayer1AbsorSolar & Exterior Slab Layer 1 Attributes & 0.7 & $\%$ \\
\hline ExtSlabLayer1AbsorVis & Exterior Slab Layer 1 Attributes & 0.7 & $\%$ \\
\hline ExtSlabLayer2Name & Exterior Slab Layer 2 Attributes & CP02 CARPET PAD & N/A \\
\hline ExtSlabLayer2Roughness & Exterior Slab Layer 2 Attributes & VeryRough & N/A \\
\hline ExtSlabLayer2ThermalRes & Exterior Slab Layer 2 Attributes & 0.21648 & $(m 2-K) / W$ \\
\hline ExtSlabLayer2AbsorThermal & Exterior Slab Layer 2 Attributes & 0.9 & $\%$ \\
\hline ExtSlabLayer2AbsorSolar & Exterior Slab Layer 2 Attributes & 0.7 & $\%$ \\
\hline ExtSlabLayer2AbsorVis & Exterior Slab Layer 2 Attributes & 0.8 & $\%$ \\
\hline FloorNumLayers & Floor Number of Layers & 3 & integer \\
\hline FloorConstDesc & Floor Description & Mass_R-10.4 ci_osedFloor & N/A \\
\hline FloorConstrutionType & Floor Type & Mass & N/A \\
\hline FloorLayer1Name & Floor Layer 1 Attributes & Floor Insulation [4] & N/A \\
\hline FloorLayer1Roughness & Floor Layer 1 Attributes & MediumRough & N/A \\
\hline FloorLayer1Thickness & Floor Layer 1 Attributes & 0.0795397 & Meter \\
\hline FloorLayer1Conductivity & Floor Layer 1 Attributes & 0.045 & $\mathrm{~W} / \mathrm{m}-\mathrm{K}$ \\
\hline FloorLayer1Density & Floor Layer 1 Attributes & 265 & $\mathrm{~kg} / \mathrm{m} 3$ \\
\hline FloorLayer1SpecificHeat & Floor Layer 1 Attributes & 836.8 & $J(k g-K)$ \\
\hline FloorLayer1AbsorThermal & Floor Layer 1 Attributes & 0.9 & $\%$ \\
\hline FloorLayer1AbsorSolar & Floor Layer 1 Attributes & 0.7 & $\%$ \\
\hline FloorLayer1AbsorVis & Floor Layer 1 Attributes & 0.7 & $\%$ \\
\hline FloorLayer2Name & Floor Layer 2 Attributes & MAT-CCO5 8 HW CONCRETE & N/A \\
\hline FloorLayer2Roughness & Floor Layer 2 Attributes & Rough & N/A \\
\hline FloorLayer2Thickness & Floor Layer 2 Attributes & 0.20321 & Meter \\
\hline FloorLayer2Conductivity & Floor Layer 2 Attributes & 1.311 & $\mathrm{~W} / \mathrm{m}-\mathrm{K}$ \\
\hline FloorLayer2Density & Floor Layer 2 Attributes & 2240 & $\mathrm{~kg} / \mathrm{m} 3$ \\
\hline
\end{tabular}

B-10 


\begin{tabular}{|c|c|c|c|}
\hline Variable & Description & Default & Units \\
\hline FloorLayer2SpecificHeat & Floor Layer 2 Attributes & 836.8 & $J(k g-K)$ \\
\hline FloorLayer2AbsorThermal & Floor Layer 2 Attributes & 0.9 & $\%$ \\
\hline FloorLayer2AbsorSolar & Floor Layer 2 Attributes & 0.7 & $\%$ \\
\hline FloorLayer2AbsorVis & Floor Layer 2 Attributes & 0.7 & $\%$ \\
\hline FloorLayer3Name & Floor Layer 3 Attributes & CP02 CARPET PAD & N/A \\
\hline FloorLayer3Roughness & Floor Layer 3 Attributes & VeryRough & N/A \\
\hline FloorLayer3ThermalRes & Floor Layer 3 Attributes & 0.21648 & $(\mathrm{~m} 2-\mathrm{K}) / \mathrm{W}$ \\
\hline FloorLayer3AbsorThermal & Floor Layer 3 Attributes & 0.9 & $\%$ \\
\hline FloorLayer3AbsorSolar & Floor Layer 3 Attributes & 0.7 & $\%$ \\
\hline FloorLayer3AbsorVis & Floor Layer 3 Attributes & 0.8 & $\%$ \\
\hline DoorDesc & Swinging Door Description & Swinging_door & N/A \\
\hline DoorType & Swinging Door Type & Swinging & N/A \\
\hline DoorLayer1Name & Swinging Door Layer 1 attributes & METAL Door Medium 18Ga_1 & N/A \\
\hline DoorLayer1Roughness & Swinging Door Layer 1 attributes & Smooth & N/A \\
\hline DoorLayer1Thickness & Swinging Door Layer 1 attributes & 0.0013106 & Meter \\
\hline DoorLayer1Conductivity & Swinging Door Layer 1 attributes & 45.3149 & $\mathrm{~W} / \mathrm{m}-\mathrm{K}$ \\
\hline DoorLayer1Density & Swinging Door Layer 1 attributes & 7833.03 & $\mathrm{~kg} / \mathrm{m} 3$ \\
\hline DoorLayer1SpecificHeat & Swinging Door Layer 1 attributes & 502.08 & $J(k g-K)$ \\
\hline DoorLayer1AbsorThermal & Swinging Door Layer 1 attributes & 0.8 & $\%$ \\
\hline DoorLayer1AbsorSolar & Swinging Door Layer 1 attributes & 0.5 & $\%$ \\
\hline DoorLayer1AbsorVis & Swinging Door Layer 1 attributes & 0.5 & $\%$ \\
\hline DoorLayer2Name & Swinging Door Layer 2 attributes & AIR & N/A \\
\hline DoorLayer2Roughness & Swinging Door Layer 2 attributes & Smooth & N/A \\
\hline DoorLayer2ThermalResistance & Swinging Door Layer 2 attributes & 0.251489 & $(\mathrm{~m} 2-\mathrm{K}) / \mathrm{W}$ \\
\hline DoorLayer2AbsorThermal & Swinging Door Layer 2 attributes & 0.8 & $\%$ \\
\hline DoorLayer2AbsorSolar & Swinging Door Layer 2 attributes & 0.5 & $\%$ \\
\hline DoorLayer2AbsorVis & Swinging Door Layer 2 attributes & 0.5 & $\%$ \\
\hline DoorLayer3Name & Swinging Door Layer 3 attributes & METAL Door Medium 18Ga_2 & N/A \\
\hline DoorLayer3Roughness & Swinging Door Layer 3 attributes & Smooth & N/A \\
\hline DoorLayer3Thickness & Swinging Door Layer 3 attributes & 0.0013106 & Meter \\
\hline DoorLayer3Conductivity & Swinging Door Layer 3 attributes & 45.3149 & $\mathrm{~W} / \mathrm{m}-\mathrm{K}$ \\
\hline DoorLayer3Density & Swinging Door Layer 3 attributes & 7833.03 & $\mathrm{~kg} / \mathrm{m} 3$ \\
\hline DoorLayer3SpecificHeat & Swinging Door Layer 3 attributes & 502.08 & $J(k g-K)$ \\
\hline DoorLayer3AbsorThermal & Swinging Door Layer 3 attributes & 0.8 & $\%$ \\
\hline DoorLayer3AbsorSolar & Swinging Door Layer 3 attributes & 0.5 & $\%$ \\
\hline DoorLayer3AbsorVis & Swinging Door Layer 3 attributes & 0.5 & $\%$ \\
\hline NSDoorDesc & Non-Swinging Door Description & Non-Swinging_NR_door & N/A \\
\hline NSDoorType & Non-Swinging Door Type & Non-Swinging & N/A \\
\hline NSDoorLayer1Name & $\begin{array}{l}\text { Non-Swinging Door Layer } 1 \\
\text { attributes }\end{array}$ & METAL Door Medium 18Ga & N/A \\
\hline
\end{tabular}




\begin{tabular}{|c|c|c|c|}
\hline Variable & Description & Default & Units \\
\hline NSDoorLayer1Roughness & $\begin{array}{c}\text { Non-Swinging Door Layer } 1 \\
\text { attributes }\end{array}$ & Smooth & N/A \\
\hline NSDoorLayer1Thickness & $\begin{array}{c}\text { Non-Swinging Door Layer } 1 \\
\text { attributes }\end{array}$ & 0.0013106 & Meter \\
\hline NSDoorLayer1Conductivity & $\begin{array}{l}\text { Non-Swinging Door Layer } 1 \\
\text { attributes }\end{array}$ & 45.3149 & $\mathrm{~W} / \mathrm{m}-\mathrm{K}$ \\
\hline NSDoorLayer1Density & $\begin{array}{c}\text { Non-Swinging Door Layer } 1 \\
\text { attributes }\end{array}$ & 7833.03 & $\mathrm{~kg} / \mathrm{m} 3$ \\
\hline NSDoorLayer1SpecificHeat & $\begin{array}{l}\text { Non-Swinging Door Layer } 1 \\
\text { attributes }\end{array}$ & 502.08 & $J(k g-K)$ \\
\hline NSDoorLayer1AbsorThermal & $\begin{array}{l}\text { Non-Swinging Door Layer } 1 \\
\text { attributes }\end{array}$ & 0.8 & $\%$ \\
\hline NSDoorLayer1AbsorSolar & $\begin{array}{l}\text { Non-Swinging Door Layer } 1 \\
\text { attributes }\end{array}$ & 0.5 & $\%$ \\
\hline NSDoorLayer1AbsorVis & $\begin{array}{l}\text { Non-Swinging Door Layer } 1 \\
\text { attributes }\end{array}$ & 0.5 & $\%$ \\
\hline NSDoorLayer2Name & $\begin{array}{c}\text { Non-Swinging Door Layer } 2 \\
\text { attributes }\end{array}$ & AIR [2] & N/A \\
\hline NSDoorLayer2Roughness & $\begin{array}{c}\text { Non-Swinging Door Layer } 2 \\
\text { attributes }\end{array}$ & Smooth & N/A \\
\hline NSDoorLayer2ThermalResistance & $\begin{array}{c}\text { Non-Swinging Door Layer } 2 \\
\text { attributes }\end{array}$ & 0.352085 & $(\mathrm{~m} 2-\mathrm{K}) / \mathrm{W}$ \\
\hline NSDoorLayer2AbsorThermal & $\begin{array}{c}\text { Non-Swinging Door Layer } 2 \\
\text { attributes }\end{array}$ & 0.8 & $\%$ \\
\hline NSDoorLayer2AbsorSolar & $\begin{array}{c}\text { Non-Swinging Door Layer } 2 \\
\text { attributes }\end{array}$ & 0.5 & $\%$ \\
\hline NSDoorLayer2AbsorVis & $\begin{array}{c}\text { Non-Swinging Door Layer } 2 \\
\text { attributes }\end{array}$ & 0.5 & $\%$ \\
\hline NSDoorLayer3Name & $\begin{array}{c}\text { Non-Swinging Door Layer } 3 \\
\text { attributes }\end{array}$ & METAL Door Medium 18Ga_2 & N/A \\
\hline NSDoorLayer3Roughness & $\begin{array}{c}\text { Non-Swinging Door Layer } 3 \\
\text { attributes }\end{array}$ & Smooth & N/A \\
\hline NSDoorLayer3Thickness & $\begin{array}{c}\text { Non-Swinging Door Layer } 3 \\
\text { attributes }\end{array}$ & 0.0013106 & Meter \\
\hline NSDoorLayer3Conductivity & $\begin{array}{l}\text { Non-Swinging Door Layer } 3 \\
\text { attributes }\end{array}$ & 45.3149 & $\mathrm{~W} / \mathrm{m}-\mathrm{K}$ \\
\hline NSDoorLayer3Density & $\begin{array}{l}\text { Non-Swinging Door Layer } 3 \\
\text { attributes }\end{array}$ & 7833.03 & $\mathrm{~kg} / \mathrm{m} 3$ \\
\hline NSDoorLayer3SpecificHeat & $\begin{array}{c}\text { Non-Swinging Door Layer } 3 \\
\text { attributes } \\
\end{array}$ & 502.08 & $J(k g-K)$ \\
\hline NSDoorLayer3AbsorThermal & $\begin{array}{l}\text { Non-Swinging Door Layer } 3 \\
\text { attributes }\end{array}$ & 0.8 & $\%$ \\
\hline NSDoorLayer3AbsorSolar & $\begin{array}{c}\text { Non-Swinging Door Layer } 3 \\
\text { attributes }\end{array}$ & 0.5 & $\%$ \\
\hline NSDoorLayer3AbsorVis & $\begin{array}{l}\text { Non-Swinging Door Layer } 3 \\
\text { attributes }\end{array}$ & 0.5 & $\%$ \\
\hline NumFloors & Number of stories in building & 1 & integer \\
\hline PerimDepth & depth of the perimeter zone & 4.57 & Meter \\
\hline FloortoFloorHeight & $\begin{array}{l}\text { total height of each story (including } \\
\text { slab thickness) }\end{array}$ & 3.8 & Meter \\
\hline PlenumHeight & $\begin{array}{l}\text { height form floor level to drop } \\
\text { ceiling }\end{array}$ & 3 & Meter \\
\hline Rotation & rotation of building in degrees & 0 & degrees \\
\hline
\end{tabular}

\section{B-12}




\begin{tabular}{|c|c|c|c|}
\hline Variable & Description & Default & Units \\
\hline UseVirtualPlenum & $\begin{array}{c}\text { set to true if building has drop } \\
\text { ceiling }\end{array}$ & 'true' & N/A \\
\hline FootprintType & $\begin{array}{l}\text { description of the shape of the } \\
\text { building foot print, see Appendix C }\end{array}$ & Rectangle & N/A \\
\hline AspectRatio & $\begin{array}{l}\text { aspect ration of building, only } \\
\text { applies to foot print shape of } \\
\text { rectangle }\end{array}$ & 2 & Ratio \\
\hline ZoneLayout & $\begin{array}{c}\text { zone configuration, either } \\
\text { perimeter and core or minimum } \\
\text { zones }\end{array}$ & Perimeter and Core & N/A \\
\hline Length1 & $\begin{array}{l}\text { Key foot print dimension, see } \\
\text { Appendix C }\end{array}$ & 40 & Meter \\
\hline Width1 & $\begin{array}{l}\text { Key foot print dimension, see } \\
\text { Appendix C }\end{array}$ & 20 & Meter \\
\hline Length2 & $\begin{array}{l}\text { Key foot print dimension, see } \\
\text { Appendix C }\end{array}$ & 15 & Meter \\
\hline Width2 & $\begin{array}{l}\text { Key foot print dimension, see } \\
\text { Appendix C }\end{array}$ & 5 & Meter \\
\hline End1 & $\begin{array}{l}\text { Key foot print dimension, see } \\
\text { Appendix C }\end{array}$ & 20 & Meter \\
\hline End2 & $\begin{array}{l}\text { Key foot print dimension, see } \\
\text { Appendix C }\end{array}$ & 20 & Meter \\
\hline Offset1 & $\begin{array}{c}\text { Key foot print dimension, see } \\
\text { Appendix C }\end{array}$ & 15 & Meter \\
\hline Offset2 & $\begin{array}{l}\text { Key foot print dimension, see } \\
\text { Appendix C }\end{array}$ & 15 & Meter \\
\hline Offset3 & $\begin{array}{l}\text { Key foot print dimension, see } \\
\text { Appendix C }\end{array}$ & 0 & Meter \\
\hline HasDayLi & Defines if a building has daylighting & No & N/A \\
\hline DayLiContType & $\begin{array}{c}\text { defines the control type for } \\
\text { daylighting }\end{array}$ & Stepped & N/A \\
\hline DayLiContSetPt & daylighting setpoint & 398 & lux \\
\hline DayLiNumSteps & $\begin{array}{l}\text { Number of steps in daylighting } \\
\text { control }\end{array}$ & 3 & integer \\
\hline DayLiNumSensors & Number of daylighting sensors & 1 & integer \\
\hline DayLiMinInputFr & $\begin{array}{l}\text { minimum daylighting fixture input } \\
\text { power fraction }\end{array}$ & 0.3 & $\%$ \\
\hline DayLiMinOutputFr & $\begin{array}{l}\text { minimum daylighting fixture output } \\
\text { fraction }\end{array}$ & 0.2 & $\%$ \\
\hline SWinWallAreaFraction & South window wall area fraction & 0.4 & $\%$ \\
\hline SWinSillHeight & south window sill height & 1.1 & Meter \\
\hline SWinEdgeOffSet & $\begin{array}{l}\text { distance of south window form } \\
\text { edge of building }\end{array}$ & 0.05 & Meter \\
\hline SWinType & South window geometry style & Banded & N/A \\
\hline SOHOffset & South over hang offset & 0 & Meter \\
\hline SOHDepth & South over hang depth & 0 & Meter \\
\hline SFinOffset & South fin offset & 0 & Meter \\
\hline SFinDepth & South fin depth & 0 & Meter \\
\hline EWinWallAreaFraction & South window wall area fraction & 0.4 & $\%$ \\
\hline
\end{tabular}




\begin{tabular}{|c|c|c|c|}
\hline Variable & Description & Default & Units \\
\hline EWinSillHeight & south window sill height & 1.1 & Meter \\
\hline EWinEdgeOffSet & $\begin{array}{l}\text { distance of south window form } \\
\text { edge of building }\end{array}$ & 0.05 & Meter \\
\hline EWinType & South window geometry style & Banded & N/A \\
\hline EOHOffset & South over hang offset & 0 & Meter \\
\hline EOHDepth & South over hang depth & 0 & Meter \\
\hline EFinOffset & South fin offset & 0 & Meter \\
\hline EFinDepth & South fin depth & 0 & Meter \\
\hline NWinWallAreaFraction & South window wall area fraction & 0.4 & $\%$ \\
\hline NWinSillHeight & south window sill height & 1.1 & Meter \\
\hline NWinEdgeOffSet & $\begin{array}{c}\text { distance of south window form } \\
\text { edge of building }\end{array}$ & 0.05 & Meter \\
\hline NWinType & South window geometry style & Banded & N/A \\
\hline NOHOffset & South over hang offset & 0 & Meter \\
\hline NOHDepth & South over hang depth & 0 & Meter \\
\hline NFinOffset & South fin offset & 0 & Meter \\
\hline NFinDepth & South fin depth & 0 & Meter \\
\hline WWinWallAreaFraction & South window wall area fraction & 0.4 & $\%$ \\
\hline WWinSillHeight & south window sill height & 1.1 & Meter \\
\hline WWinEdgeOffSet & $\begin{array}{c}\text { distance of south window form } \\
\text { edge of building }\end{array}$ & 0.05 & Meter \\
\hline WWinType & South window geometry style & Banded & N/A \\
\hline WOHOffset & South over hang offset & 0 & Meter \\
\hline WOHDepth & South over hang depth & 0 & Meter \\
\hline WFinOffset & South fin offset & 0 & Meter \\
\hline WFinDepth & South fin depth & 0 & Meter \\
\hline PeopleDensity & Density of occupants & 3.91 & Person/100m 2 \\
\hline LiPowerDensity & lighting power density & 10.76 & $\mathrm{~W} / \mathrm{m} 2$ \\
\hline LiFractionRadiant & $\begin{array}{c}\text { fraction of light energy that is } \\
\text { radiant }\end{array}$ & 0.7 & $\%$ \\
\hline LiFractionReturn & $\begin{array}{l}\text { fraction of light energy that is } \\
\text { added to return air flow }\end{array}$ & 0 & $\%$ \\
\hline Equip1EquipmentName & $\begin{array}{c}\text { Name of miscellaneous equipment } \\
1 \\
\end{array}$ & PlugMisc1 & N/A \\
\hline Equip1EquipmentType & Fuel of miscellaneous equipment 1 & Electric & N/A \\
\hline Equip1PowerDensity & $\begin{array}{c}\text { Power density of miscellaneous } \\
\text { equipment } 1\end{array}$ & 8.07 & $\mathrm{~W} / \mathrm{m} 2$ \\
\hline Equip1RadiantFraction & Fuel of miscellaneous equipment 1 & 0.5 & $\%$ \\
\hline Equip1LatentFraction & $\begin{array}{l}\text { radiant fraction of energy for } \\
\text { miscellaneous equipment } 1\end{array}$ & 0 & $\%$ \\
\hline Equip1LostFraction & $\begin{array}{c}\text { fraction of energy not going to } \\
\text { space for miscellaneous equipment } \\
1\end{array}$ & 0 & $\%$ \\
\hline
\end{tabular}




\begin{tabular}{|c|c|c|c|}
\hline Variable & Description & Default & Units \\
\hline Equip2EquipmentName & $\begin{array}{l}\text { Name of miscellaneous equipment } \\
1\end{array}$ & PlugMisc2 & $\mathrm{N} / \mathrm{A}$ \\
\hline Equip2EquipmentType & Fuel of miscellaneous equipment 1 & gas & N/A \\
\hline Equip2PowerDensity & $\begin{array}{c}\text { Power density of miscellaneous } \\
\text { equipment } 1 \\
\end{array}$ & 0 & $\mathrm{~W} / \mathrm{m} 2$ \\
\hline Equip2RadiantFraction & Fuel of miscellaneous equipment 1 & 0 & $\%$ \\
\hline Equip2LatentFraction & $\begin{array}{l}\text { radiant fraction of energy for } \\
\text { miscellaneous equipment } 1\end{array}$ & 0 & $\%$ \\
\hline Equip2LostFraction & $\begin{array}{c}\text { fraction of energy not going to } \\
\text { space for miscellaneous equipment } \\
1\end{array}$ & 0.7 & $\%$ \\
\hline InfiltrationRate & $\begin{array}{l}\text { rate that outdoor air enters building } \\
\text { in an uncontrolled manner }\end{array}$ & 0.3 & $\mathrm{ACH}$ \\
\hline TypicalHoursPerDay & $\begin{array}{l}\text { number of hours that the buildings } \\
\text { is typically occupied }\end{array}$ & 8 & hours \\
\hline OAPerArea & $\begin{array}{l}\text { Volume outdoor air brought in by } \\
\text { HVAC system per } \mathrm{m} 2\end{array}$ & 0 & $(\mathrm{~m} 3 / \mathrm{s})$ per $\mathrm{m} 2$ \\
\hline OAPerPerson & $\begin{array}{l}\text { Volume outdoor air brought in by } \\
\text { HVAC system per person }\end{array}$ & 0 & $\begin{array}{l}(\mathrm{m} 3 / \mathrm{s}) \text { per } \\
\text { person }\end{array}$ \\
\hline HVACSystemType & Type of HVAC system to model & PSZ-AC & N/A \\
\hline ZoneDistType & zone HVAC distribution mechanism & $\mathrm{AHU}$ & N/A \\
\hline ReheatCoilType & fuel for reheat coil & HOT WATER & N/A \\
\hline ReheatCoilEfficiency & efficiency of reheat coil & 0 & $\%$ \\
\hline MinFlowFraction & $\begin{array}{l}\text { minimum flow of supply air for VAV } \\
\text { systems }\end{array}$ & 0.3 & $\%$ \\
\hline UseNightCycle & 0 & TRUE & Boolean \\
\hline SATManagerType & Supply air temperature reset type & OutsideAir & N/A \\
\hline UseEconomizer & $\begin{array}{c}\text { Determines if economizer is used or } \\
\text { not }\end{array}$ & FALSE & Boolean \\
\hline MotorizedDamper & & FALSE & Boolean \\
\hline UseControllerMechVent & & FALSE & Boolean \\
\hline CoolingCoilFuel & Fuel for cooling coil & Water & N/A \\
\hline HeatingCoilFuel & Fuel for heating coil & Water & N/A \\
\hline SysFanType & defines system fan type & Variable & N/A \\
\hline SysFanEfficiency & defines system fan efficiency & 0.6 & $\%$ \\
\hline SysFanPressureDrop & defines system fan pressure drop & 500 & pa \\
\hline HeatingPumpType & $\begin{array}{l}\text { defines type of circulation pump for } \\
\text { heating system }\end{array}$ & Variable & N/A \\
\hline HeatingPumpEfficiency & $\begin{array}{l}\text { defines efficiency of heating system } \\
\text { pump }\end{array}$ & 0.9 & $\%$ \\
\hline HeatingPumpHead & $\begin{array}{l}\text { defines head of heating system } \\
\text { pump }\end{array}$ & 15000 & pa \\
\hline BoilerEfficiency & Defines boiler efficiency & 0.8 & $\%$ \\
\hline BiolerFuel & Defines boiler fuel & NaturalGas & N/A \\
\hline
\end{tabular}

\section{B-15}




\begin{tabular}{|c|c|c|c|}
\hline Variable & Description & Default & Units \\
\hline CoolingPumpType & $\begin{array}{l}\text { defines type of circulation pump for } \\
\text { cooling system }\end{array}$ & Variable & N/A \\
\hline CoolingPumpEfficiency & $\begin{array}{c}\text { defines efficiency of cooling system } \\
\text { pump }\end{array}$ & 0.9 & $\%$ \\
\hline CoolingPumpHead & $\begin{array}{c}\text { defines head of cooling system } \\
\text { pump }\end{array}$ & 15000 & pa \\
\hline ChillerType & Type of chiller to be used & $\begin{array}{l}\text { Electrically Operated Rotary } \\
\text { Screw and Scroll }\end{array}$ & N/A \\
\hline ChillerCondenserType & $\begin{array}{c}\text { chiller condenser cooling } \\
\text { mechanism }\end{array}$ & Water Cooled & N/A \\
\hline CoolingCOP & $\begin{array}{l}\text { Coefficient of performance for the } \\
\text { cooling system }\end{array}$ & 3 & Factor \\
\hline ChillerModelType & $\begin{array}{c}\text { defining how chiller is simulated in } \\
\text { EnergyPlus }\end{array}$ & ELECTRIC DOE2 & N/A \\
\hline HeatRejectionType & $\begin{array}{l}\text { if condenser is water cooled defines } \\
\text { type of cooling }\end{array}$ & WaterCooledSingleSpeedTower & N/A \\
\hline ZoneCoolFanEfficiency & $\begin{array}{l}\text { efficiency of fan used in zone for } \\
\text { cooling system }\end{array}$ & 0.6 & $\%$ \\
\hline ZoneCoolFanPressureDrop & $\begin{array}{l}\text { pressure drop of fan used in zone } \\
\text { for cooling system }\end{array}$ & 500 & pa \\
\hline PTACOperMode & $\begin{array}{l}\text { operation mode of packaged } \\
\text { terminal air conditioner }\end{array}$ & CyclingFanAndCompressor & N/A \\
\hline ZoneFanEfficiency & $\begin{array}{l}\text { efficiency of fan used in zone for } \\
\text { heating system }\end{array}$ & 0.6 & $\%$ \\
\hline ZoneFanPressureDrop & $\begin{array}{l}\text { pressure drop of fan used in zone } \\
\text { for heating system }\end{array}$ & 300 & pa \\
\hline HeatCoilEfficiency & efficiency of heating coil & 0.8 & $\%$ \\
\hline HeatingCOP & $\begin{array}{l}\text { coefficient of performance for heat } \\
\text { pump }\end{array}$ & 3 & Factor \\
\hline HPOperMode & operation mode of heat pump & CyclingFanAndCompressor & N/A \\
\hline HeatCoilType & Type of heating coil & Water & N/A \\
\hline PTHPOperMode & $\begin{array}{c}\text { operation mode of packaged } \\
\text { terminal heat pump }\end{array}$ & CyclingFanAndCompressor & N/A \\
\hline FanCoilType & function of fan coil & HeatCool & $\mathrm{N} / \mathrm{A}$ \\
\hline DHWUseRateType & $\begin{array}{c}\text { defines units for domestic hot water } \\
\text { use rate }\end{array}$ & PersonPerHour & N/A \\
\hline DHWUseRate & domestic hot water use volume & 0.001 & m3 \\
\hline DHWSupplyTemp & $\begin{array}{c}\text { domestic hot water delivery } \\
\text { temperature }\end{array}$ & 40 & C \\
\hline DHWSetPoint & $\begin{array}{l}\text { domestic hot water storage } \\
\text { temperature }\end{array}$ & 60 & C \\
\hline DHWPumpType & $\begin{array}{c}\text { domestic hot water system pump } \\
\text { type }\end{array}$ & Variable & N/A \\
\hline DHWPumpEfficiency & $\begin{array}{c}\text { domestic hot water system pump } \\
\text { efficiency }\end{array}$ & 1 & $\%$ \\
\hline DHWPumpHead & domestic hot water system pump & 179352 & pa \\
\hline
\end{tabular}

\section{B-16}




\begin{tabular}{|c|c|c|c|}
\hline Variable & Description & Default & Units \\
\hline & head & Storage Tank & N/A \\
\hline DHWHeaterType & domestic hot water heater type & Electric & N/A \\
\hline DHWHeaterFuelType & domestic hot water fuel type & 1 & m3 \\
\hline DHWHeaterEfficiency & $\begin{array}{c}\text { domestic hot water heater } \\
\text { effichcy }\end{array}$ & 0.25 & W/m2 \\
\hline DHWHeaterVolume & domestic hot water heater volume & 4500 & W/(m2-K) \\
\hline DHWHeaterCapacity & domestic hot water heater capacity & 0 & Ratio \\
\hline DHWHeaterUValue & domestic hot water heater U value & 2 & integer \\
\hline DHWHeaterAspectRatio & $\begin{array}{c}\text { domestic hot water heater aspect } \\
\text { DHWHeatio }\end{array}$ & of nodes for simulation & domestic hot water heater number \\
\hline
\end{tabular}


B-18 


\section{APPENDIX C}

Aggregate Building Simulator Allowable Footprint Shapes 



\section{Appendix C: Aggregate Building Simulator Allowable Footprint Shapes}

The ABS can handle six different footprints that cover most building configurations; these are shown below (EnergyPlus Example File Generator, 2010).

Rectangle

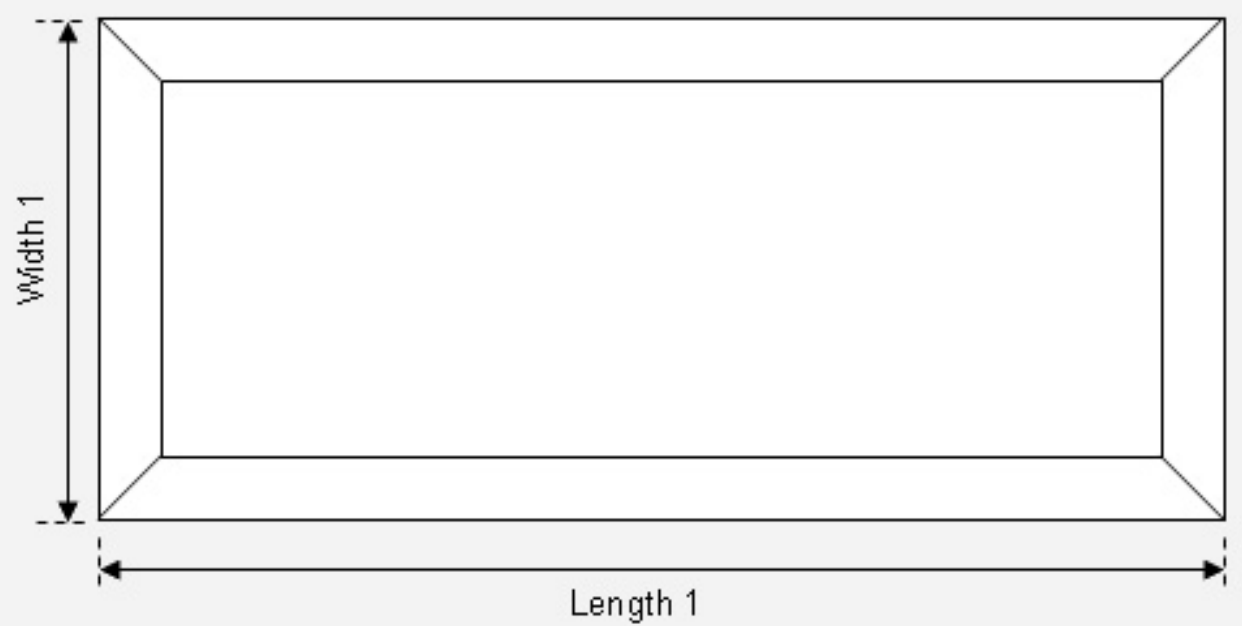

Court Yard

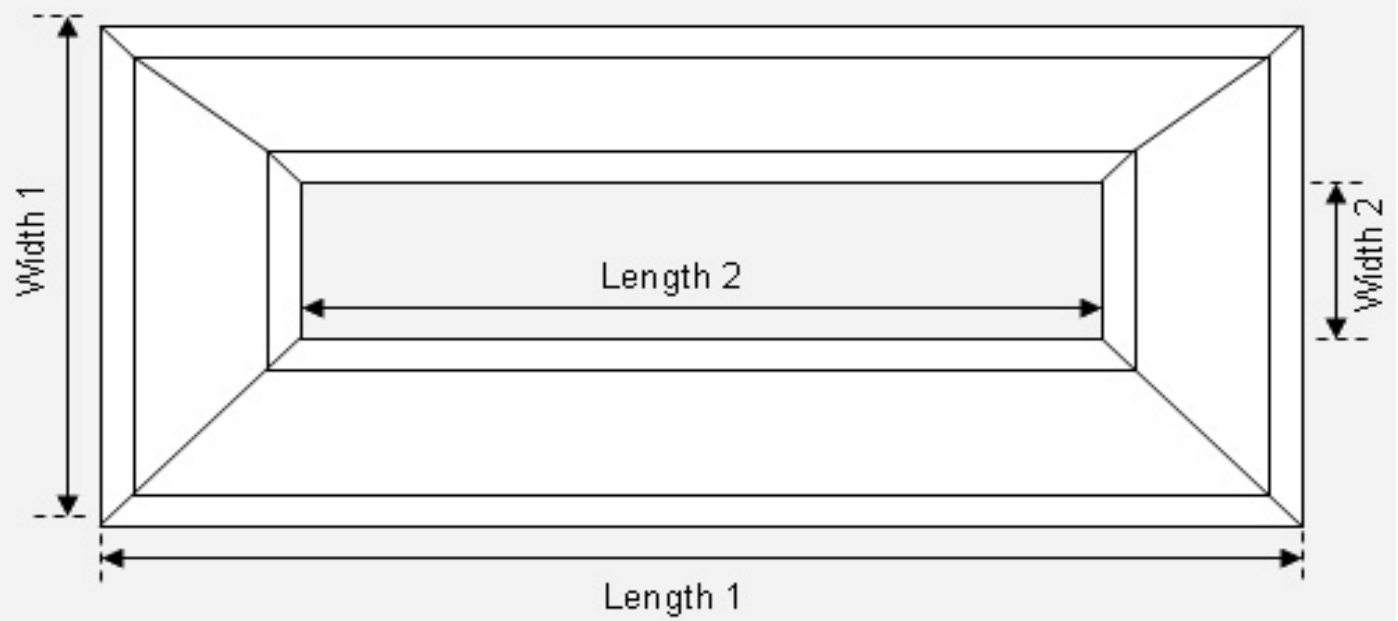

C-1 
L-Shape

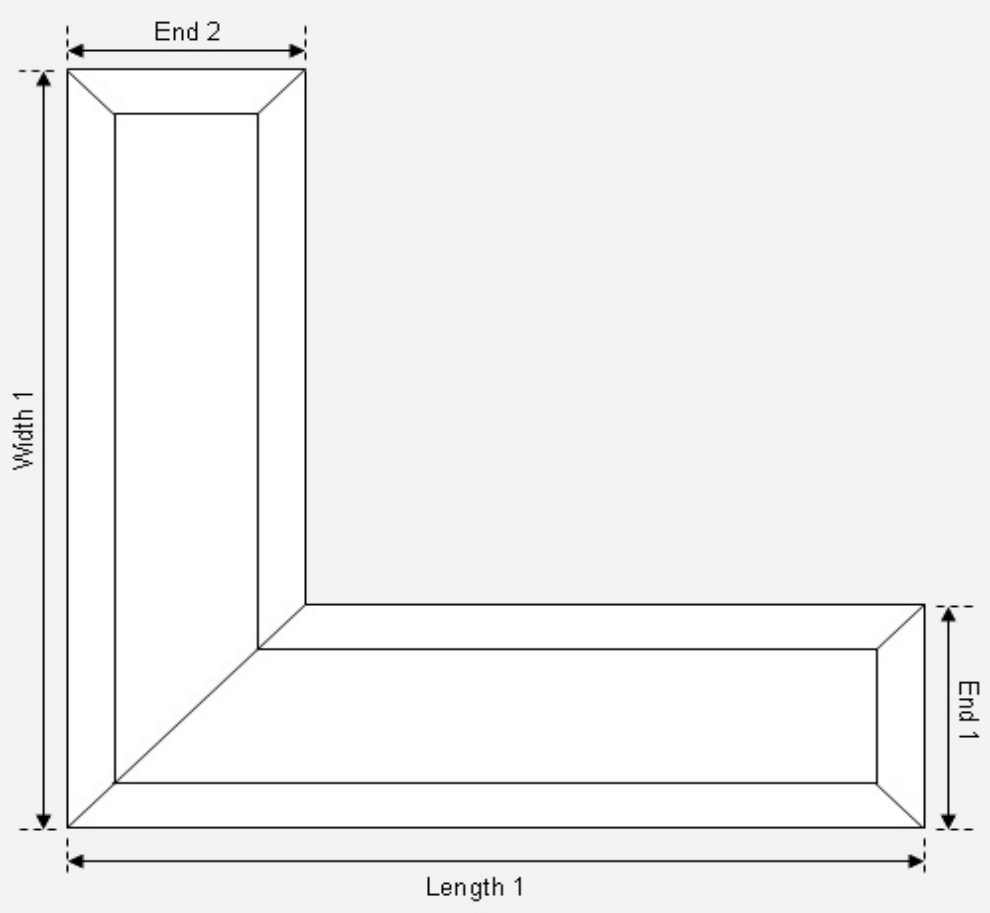

H-Shape

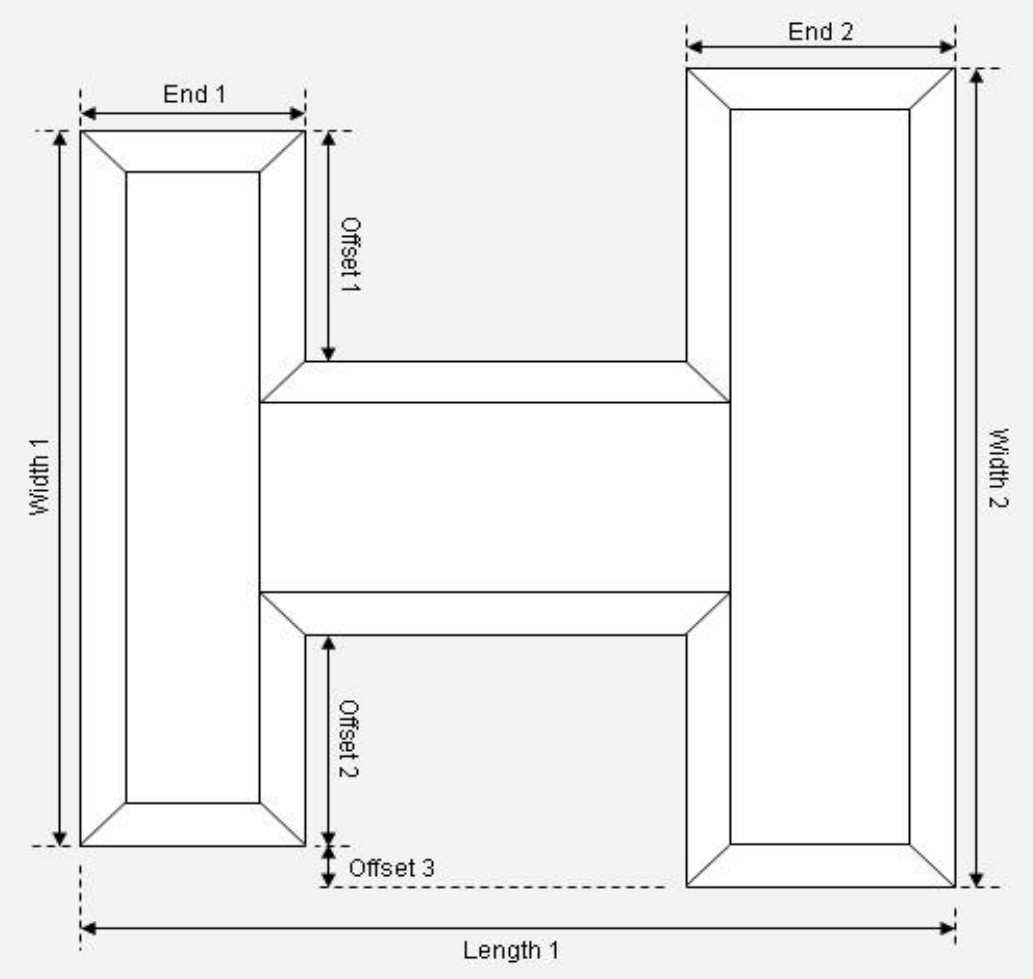

C-2 
T-shape

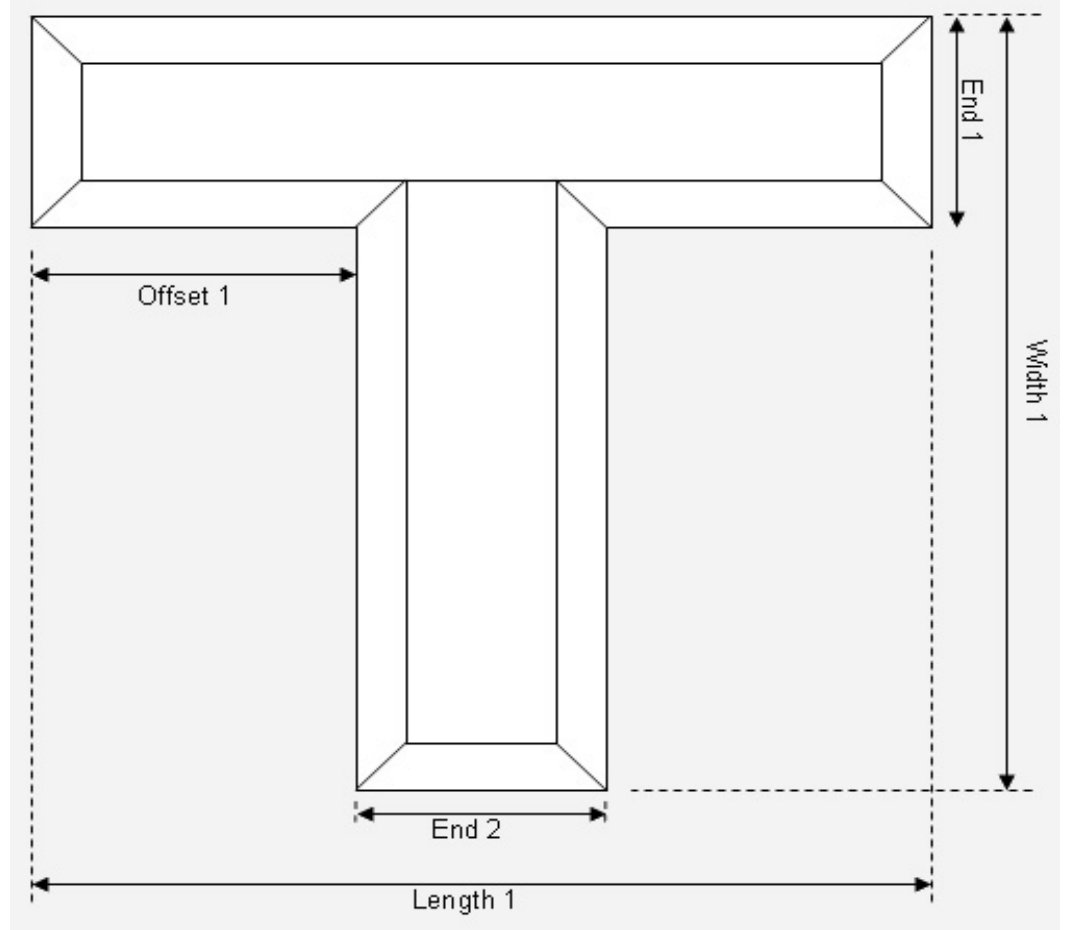

U-Shape

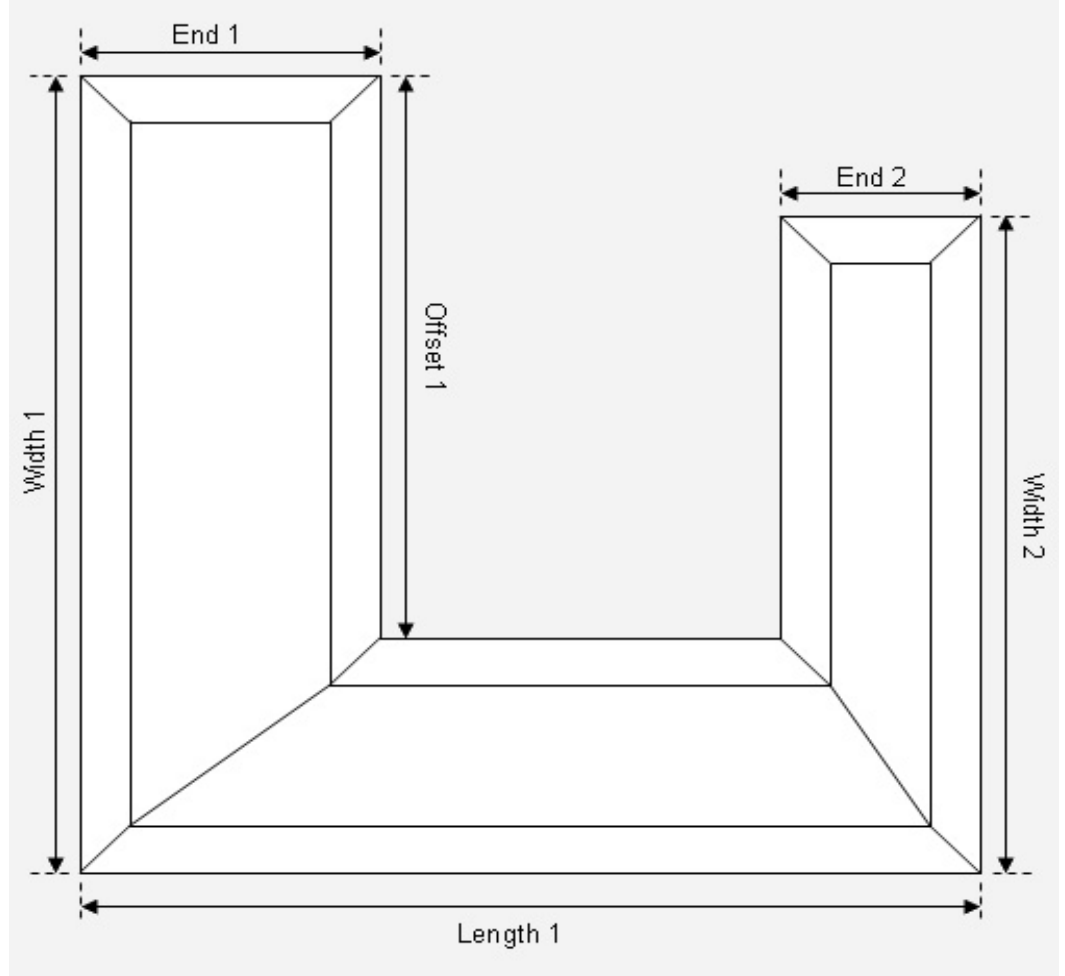

C-3 